\title{
EFNA3 as a predictor of clinical prognosis and immune checkpoint therapy efficacy in patients with lung adenocarcinoma
}

Mingming Deng ${ }^{1,2,3,4,5+}$, Run Tong ${ }^{1,3,4,5+}$, Zhe Zhang ${ }^{6}$, Tao Wang ${ }^{7}$, Chaonan Liang ${ }^{8}$, Xiaoming Zhou ${ }^{9}$ and Gang Hou $\mathrm{H}^{1,2,3,4,5^{*}}$ (D)

\begin{abstract}
Background: Ephrin receptors (Eph) and their ligands, called ephrins, function in various disease processes. However, the expression level and prognostic value of Eph/ephrins in lung adenocarcinoma (LUAD) are still unclear.

Methods: The Oncomine and GEPIA databases were used to explore the differential expression of Eph/ephrins in LUAD. Kaplan-Meier plotter was selected to explore the prognostic value of Eph/ephrins. The cBioPortal database was used to analyze the genetic variation of the EFNA3 gene. Immunohistochemistry was used to analyze the expression level and clinical value of ephrin-A3 protein in clinical LUAD tissue. Weighted coexpression network analysis (WGCNA) and gene set enrichment analysis (GSEA) identified the potential regulatory mechanism of EFNA3. CCK-8 assays and colony-forming experiments were used to investigate whether EFNA3 can regulate cell proliferation ability in LUAD. Analysis of lactate, ATP, and glucose uptake levels was used to explore the effect of EFNA3 on glycolysis ability. In addition, we investigated the relationship between EFNA3 and tumor infiltrating immune cells (TIICs). Finally, the potential immunotherapy response prediction value of EFNA3 was also explored.

Results: In this study, we found that EFNA3 expression was significantly correlated with both overall survival (OS) and progression-free survival (PFS) in LUAD patients based on a comprehensive analysis of the Eph/Ephrin family. Next, the expression of the EFNA3 protein was increased in LUAD tissues and was designated an independent prognostic risk factor. Mechanistically, EFNA3 may be involved in nuclear division, synaptic function, and ion channel activity-related pathways. In vitro experiments confirmed the role of EFNA3 in promoting LUAD cells and showed that it could regulate glycolytic capacity. Moreover, EFNA3 was negatively associated with immunity, stromal infiltration, and several TIICs. Finally, EFNA3 was found to be positively related to multiple immunotherapy biomarkers.
\end{abstract}

Conclusions: In conclusion, increased EFNA3 in LUAD patients predicted worse clinical prognosis, promoted LUAD cell proliferation and glycolysis ability, and was related to immunotherapy response.

Keywords: EFNA3, Lung cancer, Prognosis, Biomarker, Immunotherapy response

*Correspondence: hougang@zryhyy.com.cn

${ }^{\dagger}$ Mingming Deng and Run Tong contributed equally to this work

${ }^{1}$ Department of Pulmonary and Critical Care Medicine, Center

of Respiratory Medicine, China-Japan Friendship Hospital, Beijing 100029, China

Full list of author information is available at the end of the article

\section{Introduction}

Lung cancer was the most commonly diagnosed cancer in 2018 , accounting for nearly $20 \%$ of cancer deaths that year [1]. Lung adenocarcinoma (LUAD) is a predominant pathologic subtype of lung cancer [2]. Despite progress in comprehensive therapies, including surgery, radiotherapy, and targeted therapy, over the past 20 years, the OS 
of LUAD patients remains poor [3, 4]. Therefore, continued exploration of the prognostic biomarkers in patients and therapeutic targets for LUAD is necessary to provide more individualized therapies that could lead to better prognoses.

Ephrin receptors (Eph) and ephrins, their membraneanchored ligands, are essential for the development and organization of multicellular organisms. Eph/ephrins have been shown to function in various disease-related processes [5-8]. Ephs are activated by binding to ephrin ligands. Ephs and ephrins are divided into two subfamilies. The first are EphA receptors (EphA1 to EphA8 and EphA10), which primarily bind to GPI-anchored ephrinA ligands (EFNA1 to EFNA5). The second are the EphB receptors (EphB1 to EphB4 and EphB6) that preferentially engage transmembrane ephrin-B ligands (EFNB1 to EFNB3) [9, 10]. Aberrant expression of Eph/ephrins has been identified in different types of human cancers. The mode of action has been implicated to affect malignant tumors through two-way signal transduction as well as interaction with other signaling systems [11]. Recent studies have shown that EphA2, Ephrin-A1, and EphrinB2 are closely implicated in the prognosis of patients with LUAD $[12,13]$. However, the expression level and prognostic value of Eph/ephrins in lung adenocarcinoma (LUAD) are still unclear.

In this study, the expression levels and prognostic value of the Eph/ephrins family in LUAD were analyzed using bioinformatics. EFNA3 was further analyzed to verify the expression levels and prognostic value in LUAD through clinical samples. Finally, the biological function and regulatory mechanism of EFNA3 were explored based on bioinformatics analysis and in vitro experiments. Taken together, these findings indicate that increased EFNA3 in LUAD patients predicted worse clinical prognosis, promoted LUAD cell proliferation and glycolysis ability, and was related to the immunotherapy response.

\section{Materials and me thods Bioinformatics analysis}

The Oncomine database (http://www.oncomine.org) is a tumor microarray database, that has collected 715 microarray data sets as well as 86,733 cancer and normal tissue sample data sets[14]. In this study, the Oncomine database was used to analyze the expression levels of the Eph/ Ephrin family in different types of cancers.

The GEPIA database [15] (http://gepia.cancer-pku.cn/) was also used to analyze Eph/Ephrin expression in the TCGA-LUAD database.

Likewise, the Kaplan-Meier plotter (http://kmplot. com) is a tool for evaluating prognostic markers for breast cancer, ovarian cancer, lung cancer, and gastric cancer [16]. In this study, the Kaplan-Meier plotter was used to analyze the prognostic value of the Eph/Ephrin family in lung adenocarcinoma. The cBioPortal database (http://www.cbioportal.org) is an open-source database of DNA copy number, DNA methylation, and mutations based on the TCGA database [17]. Here, cBioPortal was used to analyze genetic variation of the EFNA3 gene.

Gene set enrichment analysis (GSEA) was performed as previously described[18]. The EFNA3-high/low groups were divided following the median expression of EFNA3 based on the TCGA database. The MSigDB KEGG gene set was used as a reference.

\section{Patients and tissue samples}

There were 74 primary LUAD tissue samples and paired normal lung tissue samples collected from Shengjing Hospital, China Medical University. Pathological staging of patients in this study was done according to the eighth edition of the AJCC TNM staging. 31 patients with stage I, 35 patients with stage II, and 8 patients with stage IIIIV. None of these patients had undergone chemotherapy or radiotherapy before surgery. In the eighth edition of the TNM classification, patients with stage I were divided into stage IA (tumor $\leq 3 \mathrm{~cm}$, no lymph node metastasis and no distant metastasis) and stage IB (a tumor $>3 \mathrm{~cm}$ but $\leq 4 \mathrm{~cm}$, no lymph node metastasis, and no distant metastasis). In this study, 31 patients with stage I were divided into stage IA $(n=19)$ and stage IB $(n=12) .12$ patients with stage IB and 43 patients with stage II-IV received adjuvant chemotherapy as platinum-based regimens after surgery following clinical treatment guidelines. Due to tissues were collected via surgery, follow-up treatment does not affect the Ephrin-A3 expression. Clinicopathological data were obtained from medical records and pathological reports. The study was approved by the Human Ethics Review Committee of Shengjing Hospital, China Medical University, and written informed consent was obtained from all enrolled patients.

\section{Immunohistochemistry (IHC) analysis}

IHC staining was performed and IHC scores were measured as described previously [18]. Formalin-fixed, paraffin-embedded primary LUAD tissues were sectioned into $4 \mu \mathrm{m}$ thickness. IHC staining was performed according to the manufacturers' instructions (UltraSensitiveTM SP; MXB, China). In short, LUAD tissue sections were fixed and blocked using routine laboratory procedures, followed by incubation with primary antibodies of EFNA3 (1:100; Catalog\# ab89472; Abcam) overnight at $4{ }^{\circ} \mathrm{C}$. The sections were then incubated with secondary antibody at room temperature, and visualized with 3,3-diamino-benzidine tetrahydrochloride (DAB; Maixin Biotech, China). Each section was evaluated and scored independently by two pathologists. A semi-quantitative scoring system 
was used in this assay. Intensity was scored as "0" (negative), "1" (weak), "2" (moderate), and "3" (strong). We also calculated the proportion of tumor cells within each category. The proportional score was then multiplied by the staining intensity score to generate a final IHC score. The IHC scores ranged from zero (minimum) to 300 (maximum). Patients with high expression of EFNA3 was defined as detectable immunoreactions with an IHC score $>10$.

\section{Weighted co-expression network analysis (WGCNA)}

To explore the potential function of EFNA3, weight coexpression network analysis (WGCNA) was constructed. Approximately 4096 genes (according to variance) were extracted to construct WGCNA using a "WGCNA" package. The adjacency matrix was converted into the topological overlap matrix (TOM) when the power of $\beta$ was equal to $3\left(R^{2}=0.868\right)$. Similar modules were merged following a height cutoff of 0.25 . The module of highest correlation with EFNA3 expression was selected to explore its biological function through GO and KEGG analyses.

\section{Cell culture and transfection}

Human bronchial epithelial cell line HBE, the human LUAD cell lines (A549, H1299, PC9, and HCC827) were obtained from the China Infrastructure of Cell Line Resource. All the cell lines were mycoplasma negative and authenticated using short tandem repeat authentication. Cells were cultured in dulbecco's modified eagle medium (DMEM, Gibco, USA) supplemented with $10 \%$ fetal bovine serum (FBS, Gibco, USA) at $37^{\circ} \mathrm{C}$ in a humidified $5 \% \mathrm{CO}_{2}$ incubator. Small interfering RNA (siRNA) transfection was performed as previously described [18]. The siRNA sequence of EFNA3 was 5'-ATCCTCCGGTTC TTGCAGT- $3^{\prime}$. And the siRNA sequence of negative control (NC) was 5'- UUCUCCGAACGUGUCACGUTT-3'. Transfection efficiency was identified at $48 \mathrm{~h}$ after transfection by qRT-PCR and western blot analyses.

\section{RNA isolation and real-time polymerase chain reaction (PCR)}

RNA was isolated and reverse transcribed as previously described [19]. The primer sequences for amplification were as follows: forward (5'- AGTTCTCGGAGAAGT TCCAGCG-3') and reverse (5'- CAGCAGACGAAC ACCTTCATCC-3') for EFNA3 (forward: 5'-CCCGGG GAGGTAGTGACGAAAAAT-3', reverse: 5'-CGCCCG CCCGCTCCCAAGAT-3') for the $18 \mathrm{~S}$ control.

\section{Plasmid transfections}

The overexpression plasmids containing whole coding sequence of EFNA3(NCBI Reference Sequence: NM_004952.5; ATGGCGGCGGCTCCGCTGCTGCTG
CTGCTGCTGCTCGTGCCCGTGCCGCTGCTGCCG CTGCTGGCCCAAGGG CCCGGAGGGGCG CTG GGAAACCGGCATGCGGTGTACTGGAACAGCTCC AACCAGCACCTGCGGCGAGAGGGCTACACCGTG CAGGTGAACGTGAACGACTATCTGGATATTTAC TGCCCGCACTACAACAGCTCGGGGGTGGGCCCC GGGGCGGGACCGGGGCCCGGAGGCGGGGCA GAGCAGTACGTGCTGTACATGGTGAGCCGCAAC GGCTACCGCACCTGCAACGCCAGCCAGGGCTTC AAG CGC TGG GAG TGCAACCGG CCGCACGCC CCGCACAGCCCCATCAAGTTCTCGGAGAAGTTC CAGCGCTACAGCGCCTTCTCTCTGGGCTACGAG TTCCACGCCGGCCACGAGTACTACTACATCTCC ACGCCCACTCACAACCTGCACTGGAAGTGTCTG AGGATGAAGGTGTTCGTCTGCTGCGCCTCCACA TCGCACTCCGGGAGAAGCCGGTCCCCACTCTCC CCCAGTTCACCATGGGCCCCAATGTGAAGATCA ACGTGCTGGAAGACTTTGAGGGAGAGAACCCTC AGG TGCCAAGCT TGA GAAGAGCATCAGCGG GACCAGCCCCAAACGGGAACACCTCCCCTGGCC GTGGGCATCGCCTTCTTCCTCATGACGTTCTTG GCCTCCTAG) and pcDNA 3.1 vector served as the NC were purchased from HANBIO (Shanghai, China), and pcDNA3.1-EFNA3 was used to overexpressed EFNA3. Cells were seeded at $2 \times 10^{5}$ cells/well in six-well plates overnight and then transfected by Lipofectamine 2000 reagent with plasmid. After transfections for $48 \mathrm{~h}$, the expression of EFNA3 was evaluated by PCR and Western blot.

\section{CCK8 assay and colony-forming experiments}

Cells (1500 cells/well) were cultured in 96-well plates and transfected with siRNA or Plasmid. After culture for 24, 48 , or $72 \mathrm{~h}$, cells were cultured with the CCK8 solution (C0038, Beyotime, Shanghai, China) for an additional $2 \mathrm{~h}$. Cell viability was expressed as an optical density (OD) value at $450 \mathrm{~nm}$. In order to examine the effects of EFNA3 expression on human LUAD cell proliferation, A549 cells (500/well) transfected with NC-siRNA or siRNA were added to the 12-well plates. After 10 days, the number of colonies were counted.

\section{Analysis of lactate, ATP, and glucose uptake levels}

Cells $\left(2 \times 10^{5}\right.$ cells/well $)$ were cultured in 6 -well plates and transfected with siRNA or Plasmid. The culture medium and cells were collected after $48 \mathrm{~h}$. Lactate levels in the medium were determined with use of the lactate assay kit (ab65331, Abcam), ATP levels with the ATP assay kit (ab83355, Abcam) and glucose uptake levels with the glucose uptake assay kit (ab136955, Abcam). All determinations were normalized with cell numbers. 


\section{Tumor infiltrating immune analysis}

Estimate scores, stromal scores, and immune scores were obtained via ESTIMATE, which is a method that uses gene expression signatures to infer the fraction of stromal and immune cells. And, TISIDB database was used to analyze the relationships between levels of EFNA3 expression and lymphocyte, which integrates 988 immune-associated anti-tumor genes, highthroughput screening techniques, molecular profiles, and paracancerous multi-omics data, as well as various immunological data developed from 7 public databases.

\section{Immunotherapy response biomarkers}

Immunotherapy response biomarker scores of patients with LUAD from the TCGA dataset were obtained from TCIA database (The Cancer Immunome Atlas, https:// tcia.at/home) and TIDE database (Tumour Immune Dysfunction and Exclusion, http://tide.dfci.harvard. edu). Specifically, the mutation burden, number of neoantigens, number of clonal neoantigens, and number of subclonal neoantigens in patients with LUAD, obtained from the TCGA dataset, were obtained through TCIA database [20]. The TIDE score, T cell dysfunction score, and $T$ cell exclusion score of patients with LUAD from the TCGA dataset were downloaded from the TIDE database. TIDE is a computational framework construct by Jiang et al. [21] to predict immune checkpoint blockade response. The TIDE signature was validated and outperformed known immunotherapy biomarkers that could predict immunotherapy response in melanoma and lung cancer, especially in patients treated with anti-CTLA4 and anti-PD-1/PDL1 [21].

\section{Statistical analysis}

$\mathrm{R}$ (4.0) software was used for statistical analyses. Statistical comparisons were calculated using ANOVA tests, p-values $<0.05$ were considered statistically significant.

\section{Results \\ mRNA expression level of Eph/Ephrins in LUAD based on different databases}

First, the Oncomine database was used to analyze the mRNA expression levels of Eph/Ephrins in LUAD (Fig. 1A; Table 1). The following thresholds were used to analyze the data: twofold change, $\mathrm{P}$ value $<0.0001$, and a gene grade of $10 \%$.

Overall, in most datasets, EPHA1, EPHA10, EFNA3, EFNA4, EPHB1, EPHB2, EPHB3, and EFNB3 were upregulated in LUAD tissue compared with normal lung tissue. EPHA2, EPHB4, EPHB6, and EFNB1 were downregulated in LUAD tissue. To further evaluate
Eph/Ephrins expression, The Cancer Genome Atlas (TCGA) was used.

Since there were fewer normal samples in the TCGA dataset, the GTEx dataset based on the GEPIA website was included for further analysis of the differential expression of Eph/Ephrins between normal and LUAD tissue (Fig. 1B). As shown in Fig. 1C, the results were consistent with data from the Oncomine database and TCGA database. The data are as follows: EPHA10, EFNA3, EFNA4, EFNA5, EPHB1, and EPHB2 expression levels were significantly upregulated in LUAD. In addition, EFNB1, EFNB2, and EPHB6 expression levels were significantly downregulated in LUAD. Based on these results, EPHA10, EFNA3, EFNA4, EFNA5, EPHB1, $E P H B 2, E F N B 1, E F N B 2$, and $E P H B 6$ were used for the next analysis.

\section{EFNA3 acts as the most valuable prognostic biomarker in LUAD patients}

To evaluate the prognostic value of the selected Eph/ Ephrins in LUAD patients, the Kaplan-Meier plotter database was used to analyze the relationship between expression levels and OS or PFS (Figs. 2, 3). First, the relationship with OS was analyzed. In Fig. 2A-I, upregulation of EFNA3, EFNB2, EFNB1, and EPHB6 expression showed a significant correlation with poor OS in LUAD patients. In contrast, EFNA5 and EFNB2 upregulation signified a better prognosis. Conversely, EPHA10 and EFNA4 expression did not show a significant correlation with OS, so they were excluded from this study.

Next, EFNA3, EFNB2, EFNB1, EPHB6, EFNA5, and $E F N B 2$ were selected for correlation of the relationship between expression levels and PFS. As shown in Fig. 3A$\mathrm{H}$, upregulation of EFNA3, EFNB1, and EPHB2 expression showed a significant correlation with poor PFS in LUAD patients. In contrast, EFNB2 upregulation signified a better prognosis. Similarly, EPHA10, EFNA5, and EPHB6 expression did not show a significant correlation with PFS.

EFNA3 was selected next since it showed the highest HR value both in prognostic analysis for OS and PFS in LUAD patients. Patients with different stages of cancers require different therapeutic strategies and have different prognoses; therefore, subgroup analysis was applied[22]. As shown in Fig. 4A-C, higher EFNA3 expression was associated with significantly worse OS, regardless of whether the patient was diagnosed at stage 1 (Fig. 4A), stage 2 (Fig. 4B) or stage 3 (Fig. 4C).

\section{Genetic variations of EFNA3}

To determine whether upregulation of EFNA3 in LUAD tissues was caused by genetic variations, genetic variations of EFNA3 were assessed using the cBioPortal 


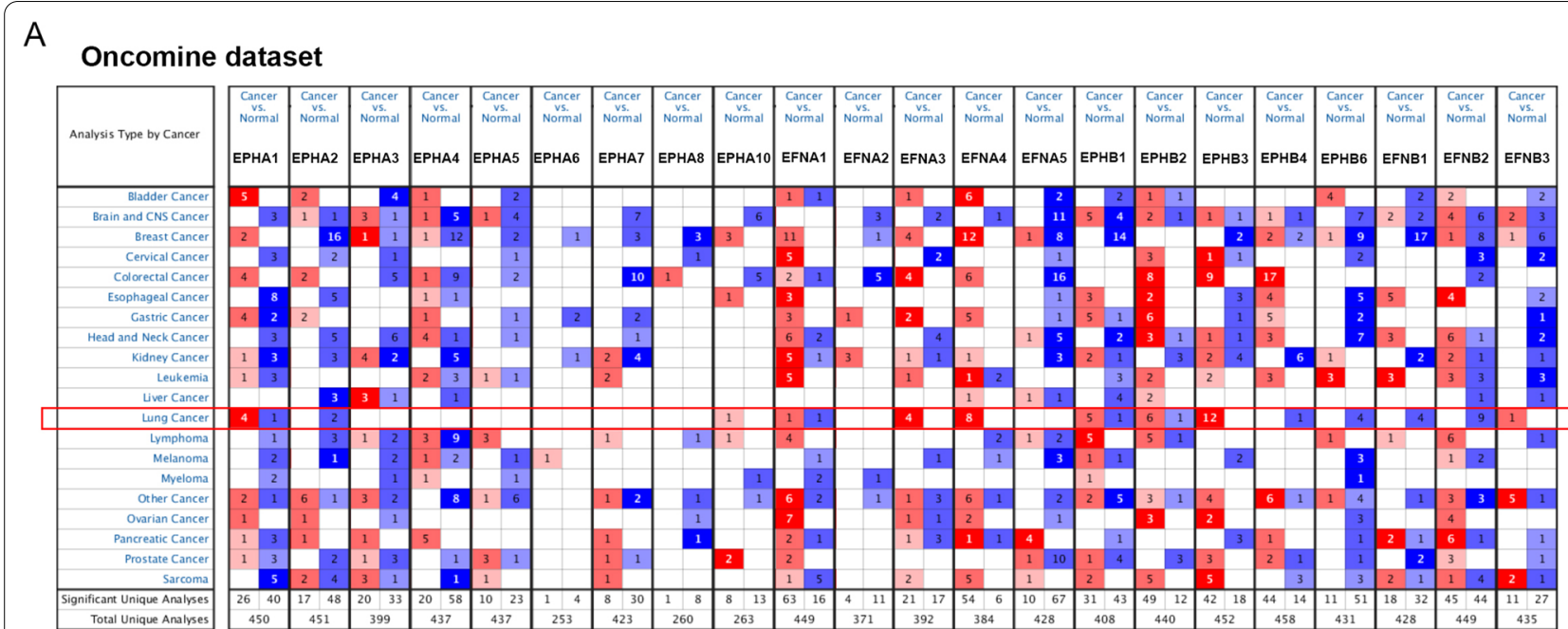

B

TCGA dataset

Type 追 Normal 追 Tumor

C

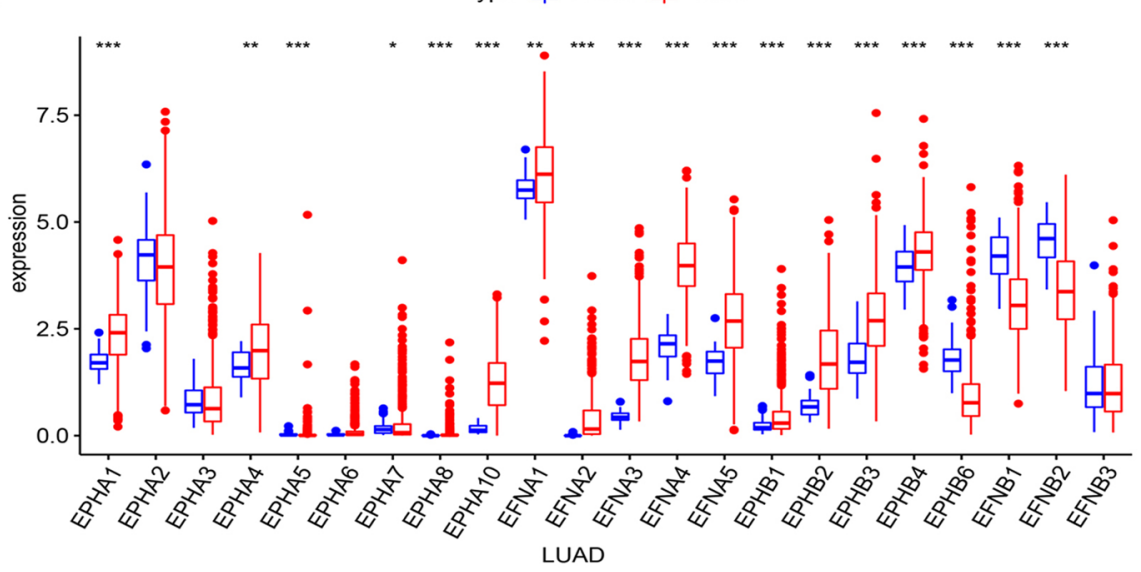

\section{TCGA+GTEx dataset}
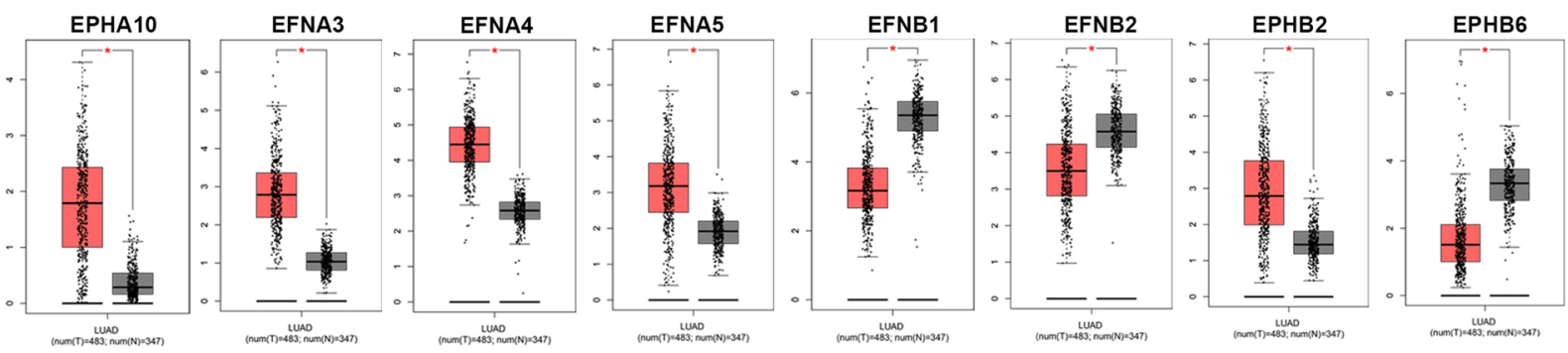

Fig. 1 Expression level of Eph/Ephrins in LUAD based on different databases $(\mathbf{A})$. The expression level of Eph/Ephrins in different types cancer based on Oncomine database; The color intensity (red or blue) is directly proportional to the significance level of upregulation or downregulation, respectively; B The expression level of Eph/Ephrins in LUAD based on TCGA dataset; C The expression level of Eph/Ephrins in LUAD based on TCGA and GTEx database, red plot and gray plot represent LUAD tissue and normal lung tissue respectively. ${ }^{*} P<0.05,{ }^{* *} P<0.01$ and ${ }^{* * *} P<0.001$

database. This database contains information on 1272 samples from five studies (Broad, Cell 2012 [23]; MSKCC, Science 2015 [24]; OncoSG, Nat Genet 2020 [25]; TCGA, Firehose Legacy [26]; TSP, Nature 2008 [27]). Genetic variations in EFNA3 showed incidence rates of $12.98 \%$ in TCGA, $7.65 \%$ in Broad, and $7.28 \%$ in OncoSG (Fig. 4D, E). Amplification was the most common type (67/67 in TCGA; $1 / 14$ in Broad; and 7/7 in OncoSG). Based on these results, amplification might be one of the main mechanisms by which EFNA3 is highly expressed in LUAD. 
Table 1 Datasets of Eph/Ephrins in lung cancer (Oncomine)

\begin{tabular}{|c|c|c|c|c|c|}
\hline Gene & Tumor (cases) & Normal (cases) & Fold change & P-value & Dataset \\
\hline \multirow[t]{5}{*}{ EPHA1 } & Lung Adenocarcinoma (86) & Lung (10) & 1.716 & $5.67 E-10$ & Beer et al \\
\hline & Lung Adenocarcinoma (58) & Lung (58) & 1.702 & $1.78 \mathrm{E}-14$ & Selamat et al \\
\hline & Squamous Cell Lung Carcinoma (13) & Lung (6) & 1.616 & 0.002 & Garber et al \\
\hline & Squamous Cell Lung Carcinoma (27) & Lung (65) & 1.878 & $2.14 \mathrm{E}-8$ & Hou et al \\
\hline & Small Cell Lung Carcinoma (6) & Lung (17) & -4.424 & $3.63 E-5$ & Bhattacharjee et al \\
\hline \multirow[t]{2}{*}{ EPHA2 } & Large Cell Lung Carcinoma (19) & Lung (65) & -3.583 & $7.45 E-13$ & Hou et al \\
\hline & Small Cell Lung Carcinoma (4) & Lung (6) & -2.827 & 0.009 & Garber et al \\
\hline EPHA10 & Lung Adenocarcinoma (226) & Lung (20) & 4.732 & $5.65 E-10$ & Okayama et al \\
\hline \multirow[t]{2}{*}{ EFNA1 } & Squamous Cell Lung Carcinoma (34) & Lung (2); Tongue (26) & 2.181 & $3.32 \mathrm{E}-10$ & Talbot et al \\
\hline & Lung Carcinoid Tumor (20) & Lung (17) & -65.441 & $5.13 E-9$ & Bhattacharjee et al \\
\hline \multirow[t]{4}{*}{ EFNA3 } & Lung Adenocarcinoma (27) & Lung (30) & 4.604 & $2.17 \mathrm{E}-8$ & Su et al \\
\hline & Squamous Cell Lung Carcinoma (27) & Lung (65) & 1.934 & $9.04 \mathrm{E}-17$ & Hou et al \\
\hline & Large Cell Lung Carcinoma (19) & Lung (65) & 1.943 & $1.88 \mathrm{E}-7$ & Hou et al \\
\hline & Lung Adenocarcinoma (86) & Lung (10) & 1.791 & $2.70 \mathrm{E}-4$ & Beer et al \\
\hline \multirow[t]{8}{*}{ EFNA4 } & Lung Adenocarcinoma (45) & Lung (65) & 2.504 & $1.35 \mathrm{E}-17$ & Hou et al \\
\hline & Squamous Cell Lung Carcinoma (27) & Lung (65) & 2.20 & $1.04 \mathrm{E}-12$ & Hou et al \\
\hline & Lung Adenocarcinoma (58) & Lung (58) & 1.722 & $1.27 \mathrm{E}-21$ & Selamat et al \\
\hline & Lung Adenocarcinoma (27) & Lung (30) & 3.720 & $2.97 \mathrm{E}-8$ & Su et al \\
\hline & Lung Adenocarcinoma (58) & Lung (49) & 2.137 & $2.98 \mathrm{E}-17$ & Landi et al \\
\hline & Squamous Cell Lung Carcinoma (5) & Lung (6) & 1.858 & $3.72 \mathrm{E}-4$ & Wachi et al \\
\hline & Lung Adenocarcinoma (226) & Lung (20) & 2.423 & $8.49 E-15$ & Okayama et al \\
\hline & Lung Adenocarcinoma (20) & Lung (19) & 2.723 & $1.27 \mathrm{E}-5$ & Stearman et al \\
\hline \multirow[t]{6}{*}{ EPHB1 } & Large Cell Lung Carcinoma (4) & Lung (6) & 2.833 & 0.001 & Garber et al \\
\hline & Lung Adenocarcinoma (36) & Lung (6) & 2.608 & $1.01 \mathrm{E}-4$ & Garber et al \\
\hline & Squamous Cell Lung Carcinoma (12) & Lung (6) & 3.360 & $1.35 E-4$ & Garber et al \\
\hline & Small Cell Lung Carcinoma (4) & Lung (6) & 4.877 & 0.010 & Garber et al \\
\hline & Squamous Cell Lung Carcinoma, Basaloid Variant (8) & Lung (390) & 1.543 & $5.08 E-4$ & TCGA \\
\hline & Lung Adenocarcinoma (38) & Lung (6) & -2.966 & $4.77 \mathrm{E}-6$ & Garber et al \\
\hline \multirow[t]{7}{*}{ EPHB2 } & Lung Adenocarcinoma (20) & Lung (19) & 1.947 & $1.15 E-7$ & Stearman et al \\
\hline & Lung Adenocarcinoma (27) & Lung (30) & 2.958 & $7.60 \mathrm{E}-7$ & Su et al \\
\hline & Lung Adenocarcinoma (45) & Lung (65) & 1.539 & $1.22 \mathrm{E}-11$ & Hou et al \\
\hline & Lung Adenocarcinoma (58) & Lung (49) & 1.594 & $6.46 \mathrm{E}-13$ & Landi et al \\
\hline & Lung Adenocarcinoma (132) & Lung (17) & 2.006 & 0.015 & Bhattacharjee et al \\
\hline & Lung Adenocarcinoma (226) & Lung (20) & 2.400 & $1.02 \mathrm{E}-10$ & Okayama et al \\
\hline & Lung Adenocarcinoma (86) & Lung (10) & -2.372 & $2.95 E-6$ & Beer et al \\
\hline \multirow[t]{12}{*}{ EPHB3 } & Squamous Cell Lung Carcinoma, Basaloid Variant (8) & Lung (390) & 1.721 & $1.21 \mathrm{E}-4$ & TCGA \\
\hline & Squamous Cell Lung Carcinoma (348) & Lung (390) & 1.560 & $1.29 E-87$ & TCGA \\
\hline & Squamous Cell Lung Carcinoma (11) & Lung (3) & 1.779 & 0.004 & Yamagata et al \\
\hline & Large Cell Lung Carcinoma (5) & Lung (3) & 1.563 & 0.014 & Yamagata et al \\
\hline & Squamous Cell Lung Carcinoma (13) & Lung (6) & 4.747 & $7.31 \mathrm{E}-5$ & Garber et al \\
\hline & Squamous Cell Lung Carcinoma (21) & Lung (17) & 5.147 & $6.27 \mathrm{E}-4$ & Bhattacharjee et al \\
\hline & Squamous Cell Lung Carcinoma (5) & Lung (6) & 1.573 & 0.001 & Wachi et al \\
\hline & Lung Adenocarcinoma (20) & Lung (19) & 1.797 & $1.29 E-4$ & Stearman et al \\
\hline & Squamous Cell Lung Carcinoma (27) & Lung (65) & 2.130 & $5.56 \mathrm{E}-10$ & Hou et al \\
\hline & Lung Adenocarcinoma (45) & Lung (65) & 1.554 & $4.02 \mathrm{E}-9$ & Hou et al \\
\hline & Large Cell Lung Carcinoma (19) & Lung (65) & 1.899 & $3.11 \mathrm{E}-5$ & Hou et al \\
\hline & Lung Adenocarcinoma (226) & Lung (20) & 1.731 & $1.24 \mathrm{E}-8$ & Okayama et al \\
\hline EPHB4 & Small Cell Lung Carcinoma (6) & Lung (17) & -6.614 & $3.49 \mathrm{E}-6$ & Bhattacharjee et al \\
\hline EPHB6 & Large Cell Lung Carcinoma (3) & Lung (6) & -1.542 & 0.003 & Garber et al \\
\hline
\end{tabular}


Table 1 (continued)

\begin{tabular}{|c|c|c|c|c|c|}
\hline Gene & Tumor (cases) & Normal (cases) & Fold change & P-value & Dataset \\
\hline & Lung Adenocarcinoma (39) & Lung (6) & -1.708 & 4.32E-4 & Garber et al \\
\hline & Small Cell Lung Carcinoma (6) & Lung (17) & -4.770 & $3.28 E-5$ & Bhattacharjee et al \\
\hline & Lung Adenocarcinoma (20) & Lung (19) & -3.105 & $1.18 \mathrm{E}-4$ & Stearman et al \\
\hline \multirow[t]{4}{*}{ EFNB1 } & Lung Adenocarcinoma (20) & Lung (19) & -1.789 & $4.73 E-8$ & Stearman et al \\
\hline & Lung Carcinoid Tumor (20) & Lung (17) & -16.859 & $8.76 \mathrm{E}-7$ & Bhattacharjee et al \\
\hline & Small Cell Lung Carcinoma (6) & Lung (17) & -3.585 & $8.46 \mathrm{E}-5$ & Bhattacharjee et al \\
\hline & Large Cell Lung Carcinoma (4) & Lung (6) & -1.592 & 0.017 & Garber et al \\
\hline \multirow[t]{9}{*}{ EFNB2 } & Lung Adenocarcinoma (132) & Lung (17) & -3.676 & $1.56 \mathrm{E}-6$ & Bhattacharjee et al \\
\hline & Lung Carcinoid Tumor (20) & Lung (17) & -9.762 & $6.10 E-10$ & Bhattacharjee et al \\
\hline & Squamous Cell Lung Carcinoma (21) & Lung (17) & -2.156 & 0.009 & Bhattacharjee et al \\
\hline & Lung Adenocarcinoma (20) & Lung (19) & -2.453 & $2.77 \mathrm{E}-6$ & Stearman et al \\
\hline & Lung Adenocarcinoma (58) & Lung (49) & -2.304 & $5.93 \mathrm{E}-17$ & Landi et al \\
\hline & Lung Adenocarcinoma (27) & Lung (30) & -2.044 & $2.96 \mathrm{E}-6$ & Su et al \\
\hline & Large Cell Lung Carcinoma (19) & Lung (65) & -3.549 & $9.56 \mathrm{E}-10$ & Hou et al \\
\hline & Squamous Cell Lung Carcinoma (5) & Lung (5) & -1.917 & 0.004 & Wachi et al \\
\hline & Lung Adenocarcinoma (58) & Lung (58) & -1.988 & $2.48 \mathrm{E}-10$ & Selamat et al \\
\hline EFNB3 & Lung Carcinoid Tumor (20) & Lung (17) & 4.500 & $1.58 E-5$ & Bhattacharjee et al \\
\hline
\end{tabular}

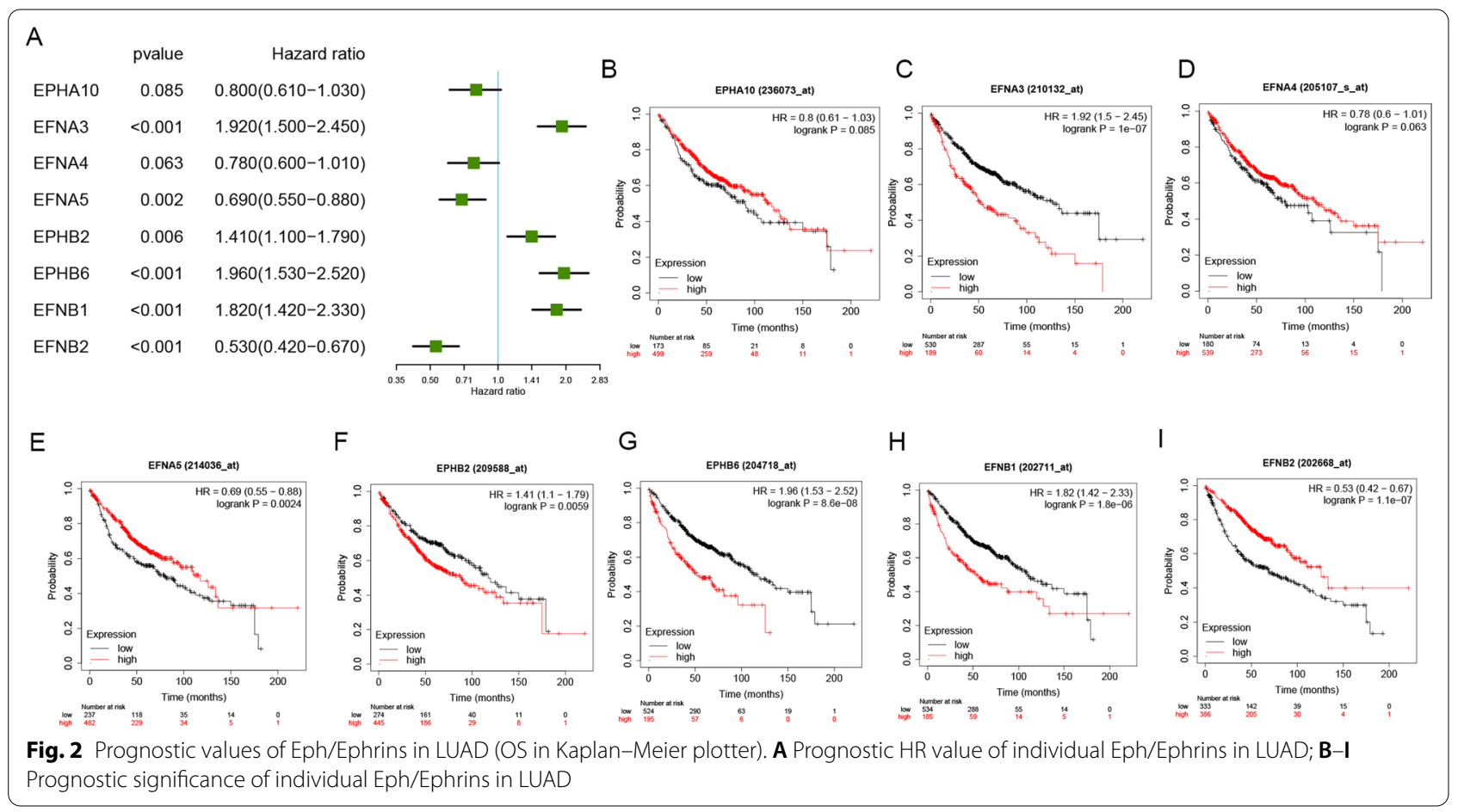

\section{The expression of the EFNA3 protein was increased in LUAD tissues and related to the prognosis of LUAD patients}

To better understand the clinical significance of the EFNA3 protein in LUAD patients, IHC was performed to investigate the expression of EFNA3 in a tissue microarray (TMA) containing 74 LUAD tissues and adjacent normal lung tissues (Fig. 5A). Compared with normal lung tissue, the expression of the EFNA3 protein was significantly increased in LUAD tissue samples (Fig. 5B). Further analysis revealed that the IHC score for EFNA3 was significantly upregulated in cases of larger tumor sizes (Fig. 5C), lymph node metastasis (Fig. 5D), and advanced TNM stage (Fig. 5E). 


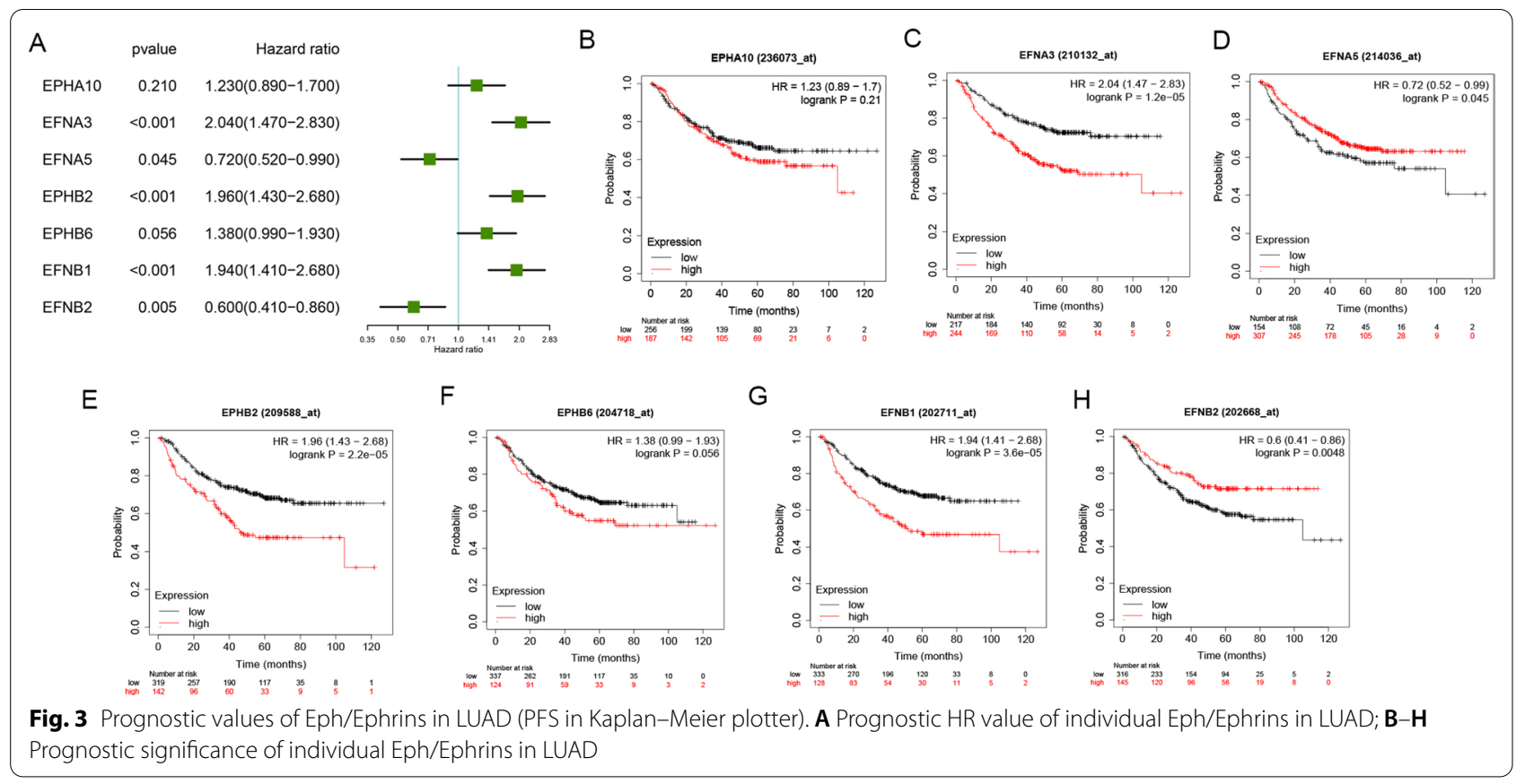

A

Stage 1

EFNA3 (210132_at)

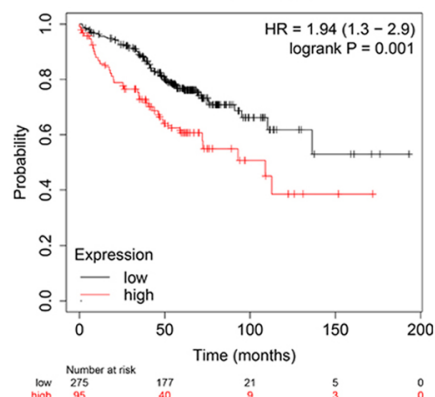

D

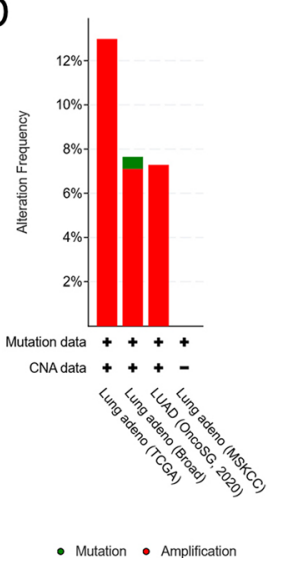

B

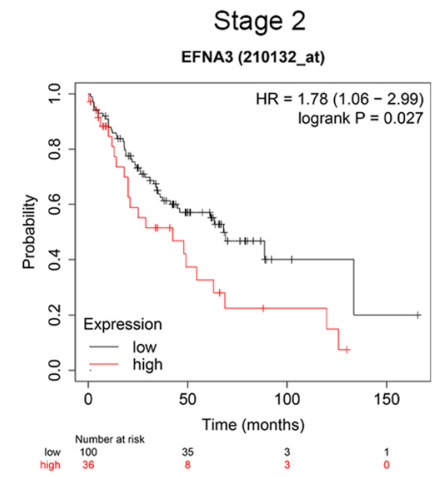

C

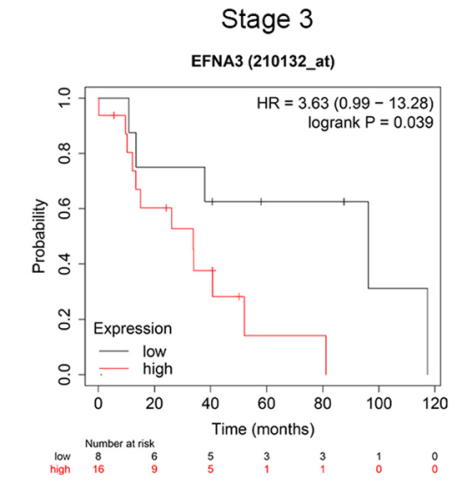

Fig. 4 Survival analyses and genetic variations of EFNA3 in LUAD. A-C Prognostic significance of EFNA3 expression in differential stage LUAD patients. D Genetic variations in the EFNA3 gene reported in different studies; E OncoPrint overview of the genetic variations in the EFNA3 gene 


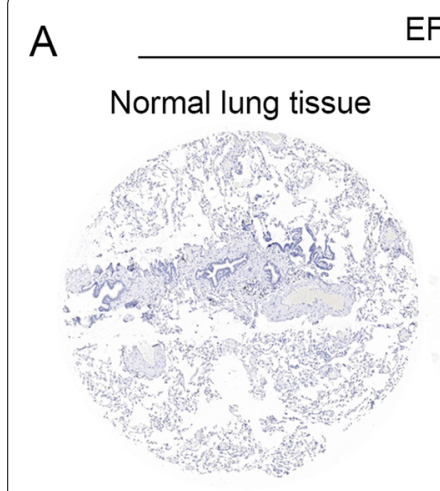

EFNA3

C

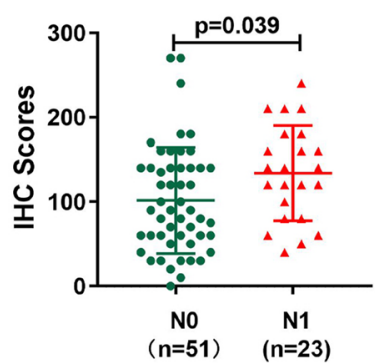

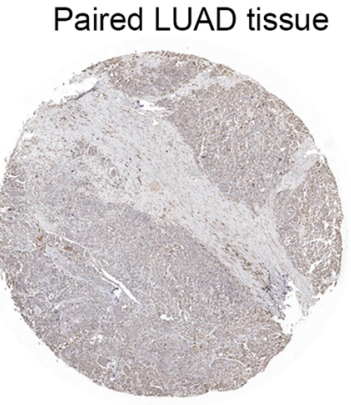

$\mathrm{D}$

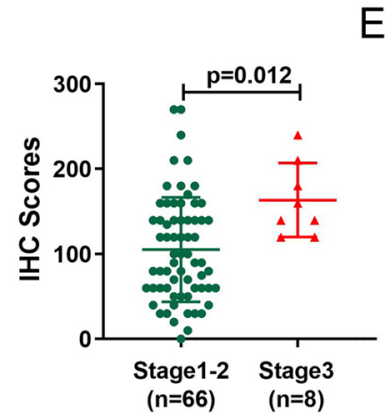

$E$
B
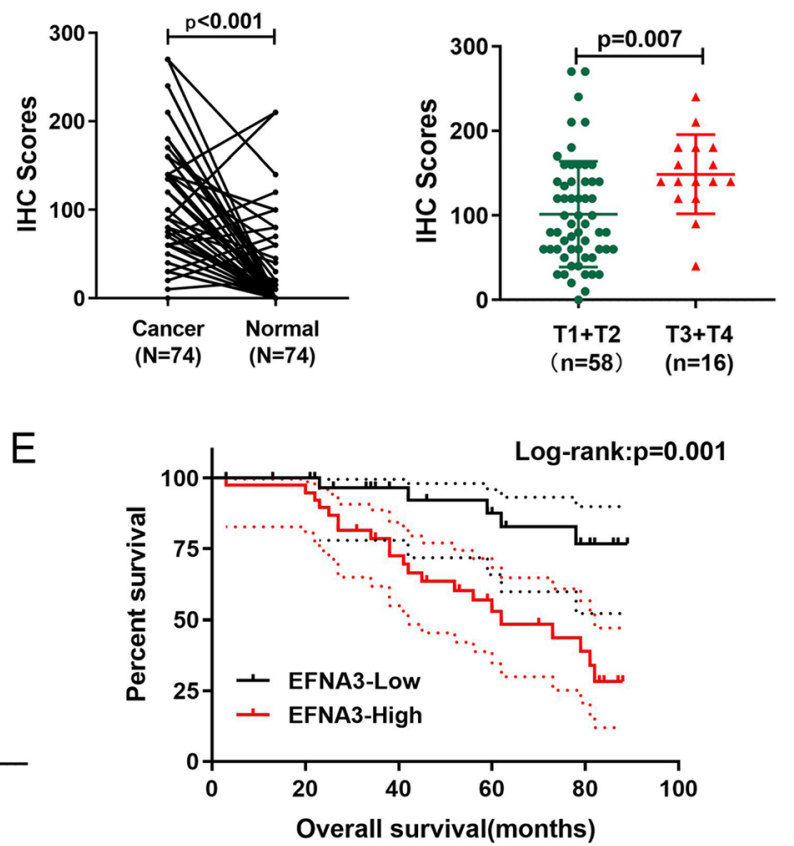

Fig. 5 Upregulation of EFNA3 in lung adenocarcinoma (LUAD) tissues and its relationship with clinicopathological features and outcomes. A Representative images of EFNA3 staining in LUAD tissue and paired normal lung tissue; $\mathbf{B}$ The IHC score of EFNA3 was significantly increased in LUAD tissues; EFNA3 staining score increased significantly with tumor size (C), lymph node metastasis (D) and advanced TNM stages (E); F KaplanMeier survival analysis and log-rank tests showed that high expression levels of EFNA3 were associated with poor prognosis $(P=0.001)$

LUAD patients were divided into two groups according to IHC scores: the EFNA3-high group and the EFNA3-low group. The chi-square test showed that EFNA3 protein expression was significantly correlated with larger tumor size $(\mathrm{P}=0.004)$, lymph node metastasis $(\mathrm{P}=0.035)$, and a higher TNM stage $(\mathrm{P}=0.002)$ (Table 2). Moreover, EFNA3-negative patients presented with shorter overall survival times than EFNA3-positive patients $(P=0.039)$ (Fig. 6F). Finally, Cox regression analysis of overall survival showed that higher EFNA3 expression was an independent prognostic risk factor $(\mathrm{HR}=3.108 ; 95 \% \mathrm{CI}=1.077-8.963 ; \mathrm{P}=0.036)$ (Table 3). Based on these results, EFNA3 could represent a new prognostic biomarker for LUAD.

\section{WGCNA and GSEA}

To identify the potential regulatory mechanism of EFNA3, the TCGA dataset was used to construct the coexpression network through WGCNA. Clinical features, including OS time, OS status, pathological parameters and EFNA3 expression, were obtained from the TCGA dataset (Fig. 6A). The parameters were established by setting the soft-threshold power to 3 (scale free $\mathrm{R} 2=0.0 .868$ ), and the height was set to 0.25 . In this study,
Table 2 Correlation between the expression of EFNA3 and clinical characteristics in LUAD patients $(n=74)$

\begin{tabular}{|c|c|c|c|c|}
\hline \multirow{2}{*}{$\begin{array}{l}\text { Clinical } \\
\text { Pathological } \\
\text { Parameters }\end{array}$} & \multirow[t]{2}{*}{ Number } & \multicolumn{2}{|c|}{ EFNA3 expression } & \multirow[t]{2}{*}{$P$ value } \\
\hline & & $\operatorname{High}(n=38)$ & $\operatorname{Low}(n=36)$ & \\
\hline Age & & & & 0.367 \\
\hline$<60$ & 29 & 13 & 16 & \\
\hline$\geq 60$ & 45 & 25 & 20 & \\
\hline Sex & & & & 0.252 \\
\hline Male & 48 & 27 & 21 & \\
\hline Female & 26 & 11 & 15 & \\
\hline Tumor size & & & & $0.004^{*}$ \\
\hline $\mathrm{T} 1$ & 15 & 5 & 10 & \\
\hline $\mathrm{T} 2$ & 43 & 19 & 24 & \\
\hline $\mathrm{T} 3+\mathrm{T} 4$ & 16 & 14 & 2 & \\
\hline LN metastasis & & & & $0.035^{*}$ \\
\hline No & 51 & 22 & 29 & \\
\hline Yes & 23 & 16 & 7 & \\
\hline TNM stage & & & & $0.002^{*}$ \\
\hline I & 31 & 10 & 21 & \\
\hline$\|$ & 35 & 20 & 15 & \\
\hline$I I I+I V$ & 8 & 8 & 0 & \\
\hline
\end{tabular}

"Significant correlation 
A

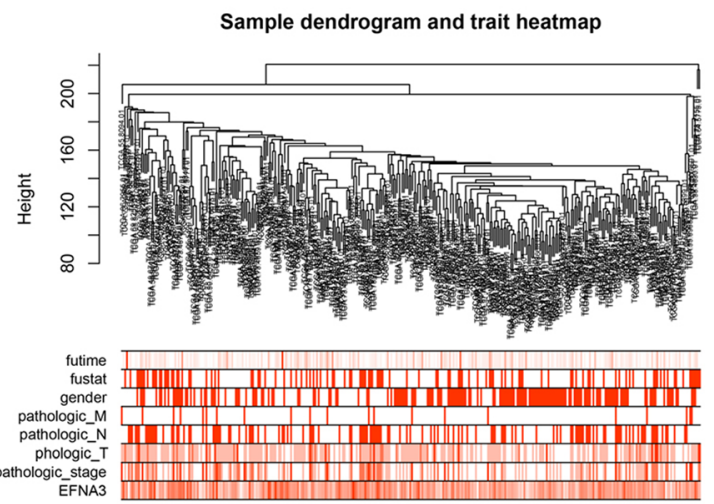

C

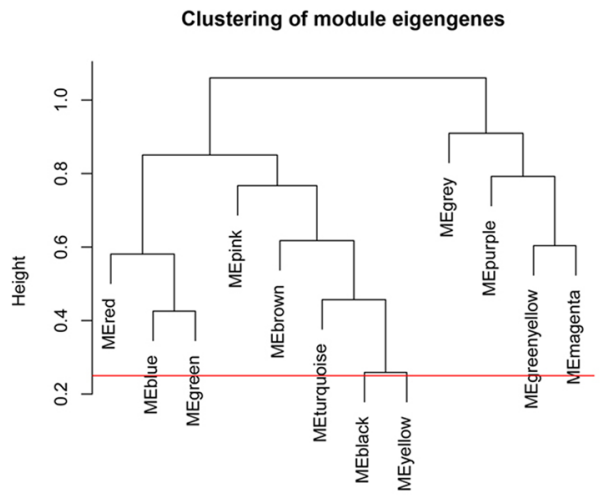

$\mathrm{B}$

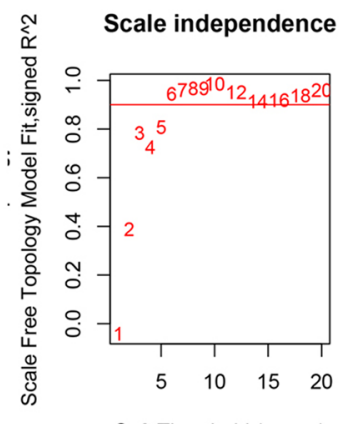

Soft Threshold (power)

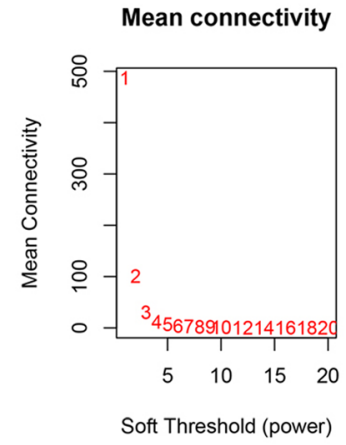

D

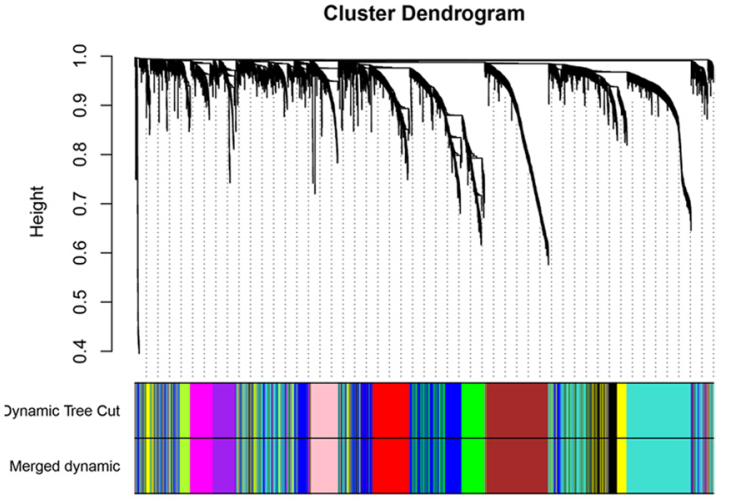

E

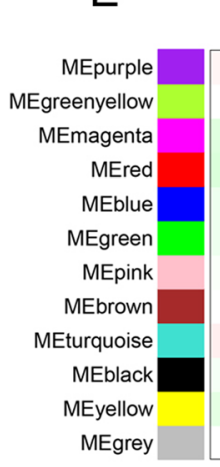

Module-trait relationships
F

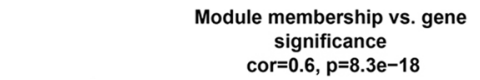
significance
cor $=0.6, p=8.3 e-18$

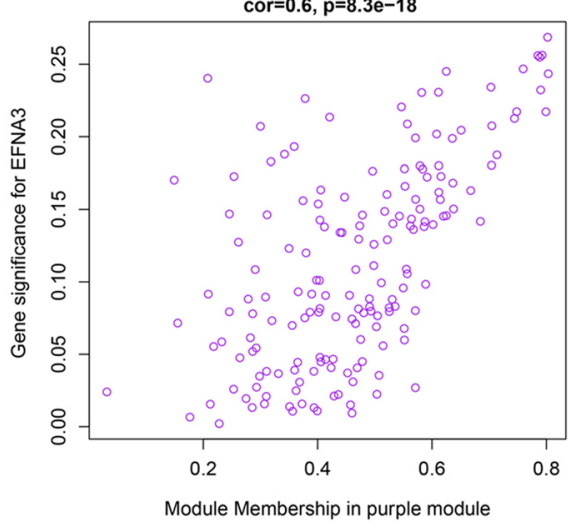

Fig. 6 Screening for key modules related to EFNA3 through WGCNA. A Sample dendrogram and trait heatmap (TCGA-LUAD); B Calculation of the scale-free fit index of various soft-thresholding powers ( $\beta$ ); C Clustering of module eigengenes. The red line indicates cut height (0.25); D Clustering dendrograms; E Correlation heatmap between module eigengenes and clinical parameters; $\mathbf{F}$ Scatter plot of purple module eigengenes

12 modules were identified (Fig. 6B-D). The association between the modules and clinical features was measured by the correlation between module eigengene (ME) values and clinical features. Data were visualized by heatmap profiles. The results showed that the purple module was the most closely correlated with EFNA3 expression (Pearson coefficient $=0.23, \mathrm{P}=1 \mathrm{E}-05$; Fig. 6E). A scatter plot of purple module eigengenes is shown in Fig. 7F. In the purple module, 168 genes were selected as hub genes for GO and KEGG analysis. 
Table 3 Cox regression analysis of overall survival in LUAD patients

\begin{tabular}{|c|c|c|c|c|c|c|}
\hline \multirow[t]{2}{*}{ Variables } & \multicolumn{3}{|c|}{ Univariate analysis } & \multicolumn{3}{|c|}{ Multivariate analysis } \\
\hline & HR & $95 \% \mathrm{Cl}$ & $P$ value & HR & $95 \% \mathrm{Cl}$ & $P$ value \\
\hline $\begin{array}{l}\text { Age (years) } \\
(\leq 60 \text { vs }>60)\end{array}$ & 1.180 & $0.529-2.629$ & 0.686 & & & \\
\hline $\begin{array}{l}\text { Gender } \\
\text { (male vs female) }\end{array}$ & 0.949 & $0.419-2.150$ & 0.900 & & & \\
\hline pT stage & 1.489 & $0.898-2.469$ & 0.123 & & & \\
\hline pN stage & 3.162 & $1.435-6.965$ & $0.004^{*}$ & 1.336 & $0.479-3.725$ & 0.580 \\
\hline pTNM stage & 2.583 & $1.505-4.432$ & $<0.001^{*}$ & 1.530 & $0.738-3.173$ & 0.253 \\
\hline EFNA3 expression & 4.272 & $1.597-11.423$ & $0.004^{*}$ & 3.108 & $1.077-8.963$ & $0.036^{*}$ \\
\hline
\end{tabular}

Factors for which $\mathrm{P}<0.05$ in univariate analysis were subsequently used for multivariate analysis

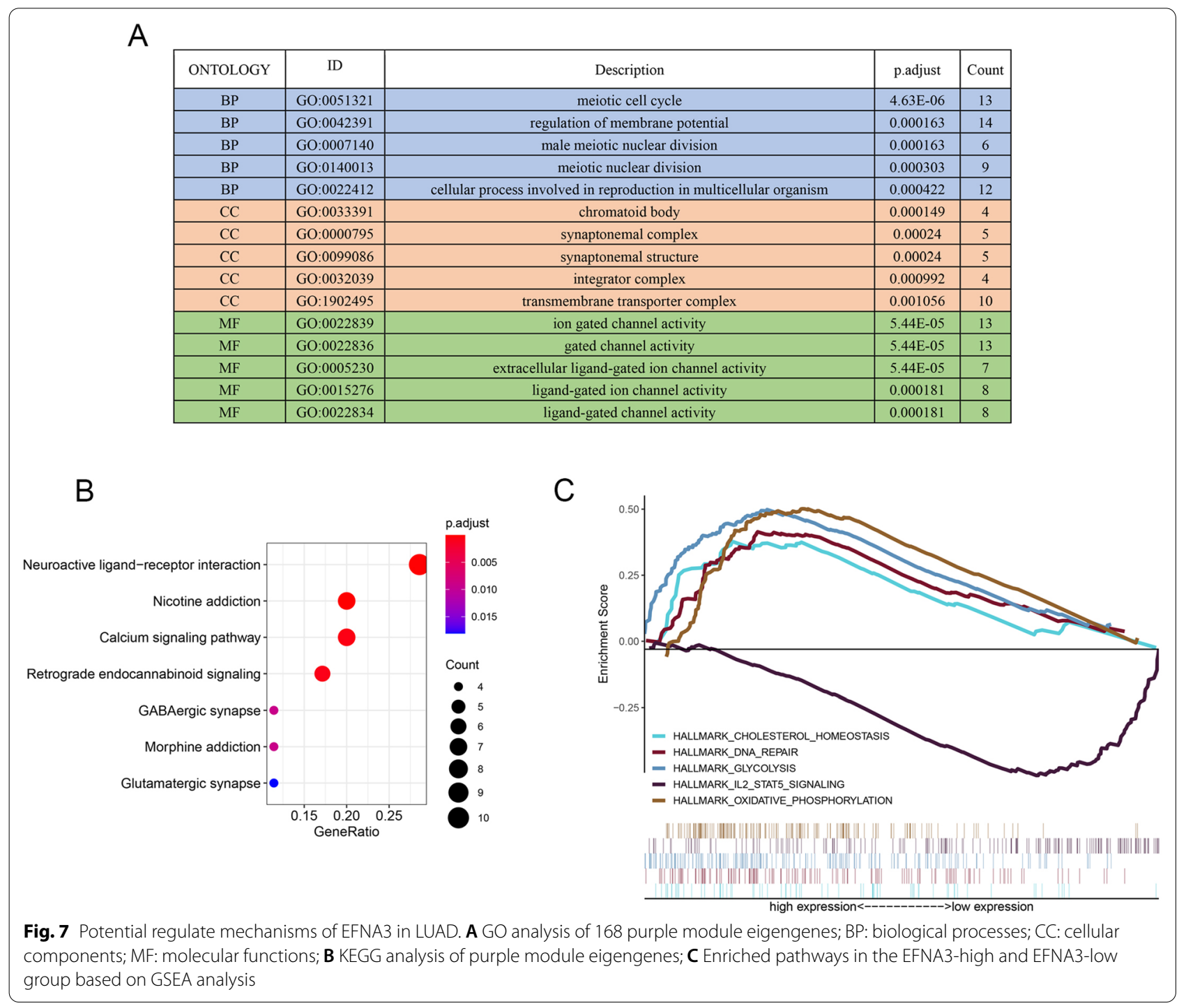


In the purple module genes, nuclear division, synaptic function, and ion channel activity-related pathways were the most frequently noted pathways in the $\mathrm{GO}$ analysis. The most enriched GO term in the biological process (BP) category was "meiotic cell cycle." In the cellular component (CC) category, "chromatoid body" was most enriched, and in the molecular function (MF) category, "ion gated channel activity" was most abundant. Furthermore, KEGG pathway enrichment analysis results contained "neuroactive ligand-receptor interaction" and "calcium signaling pathway" enrichments (Fig. 7B). Finally, GSEA was used to identify the mechanism and functional differences between the EFNA3-high expression group and the EFNA3-low expression group. As shown in Fig. 7C, "cholesterol homeostasis," "DNA repair," "glycolysis," and "oxidative phosphorylation" were enriched in the EFNA3-high expression group, whereas "IL2-STAT5 signaling" was enriched in the EFNA3-low expression group.

\section{The function of EFNA3 and its regulation of glycolysis ability}

Sustained proliferation is the most fundamental feature of cancer cells. To investigate whether EFNA3 can regulate cell proliferation ability in LUAD, we first detected the mRNA and protein expression levels of EFNA3 in human bronchial epithelial cells and lung adenocarcinoma cells. As seen in Fig. 8A, B, the mRNA and protein expression levels of EFNA3 were upregulated in LUAD cells (H1299, H1975, A549, PC9, and HCC827) compared to HBE cells. A549 cells were chosen for the next study for further investigation because of their high levels of EFNA3 expression. Knockdown of EFNA3 expression was performed using siRNA. Transfection efficiency was measured using qRT-PCR and western blot analyses (Fig. 8C). CCK-8 assays revealed that knockdown of EFNA3 significantly decreased the proliferation of A549 cells (Fig. 8D). Moreover, the EFNA3-siRNA-transfected group had significantly fewer colonies than the NCsiRNA-transfected group (Fig. 8E). Taken together, these results demonstrated that EFNA3 knockdown could decrease the proliferation ability of LUAD cells in vitro. In Fig. $8 \mathrm{C}$, we found that the glycolysis pathway was enriched in the EFNA3-high expression group. Therefore, we next explored whether EFNA3 is involved in regulating the glycolytic ability of LUAD cells. Functional colorimetric validation (Fig. 8F) showed that lactic acid production (a key metabolite of glycolysis), ATP levels and glucose uptake were both significantly decreased after EFNA3 knockdown in A549 cells.

To further validate these results, we next overexpressed EFNA3 in the HCC827 cell line, which normally expresses a low level of EFNA3. Transfection efficiency was quantified by qRT-PCR and western blotting (Fig. 8G). CCK-8 assays revealed that overexpression of EFNA3 significantly upregulated the proliferation of $\mathrm{HCC} 827$ cells (Fig. $8 \mathrm{H}$ ). Moreover, the pcDNA3.1-EFNA3-transfected group had significantly more colonies than the pcDNA3.1-NC-transfected group (Fig. 8I). Finally, lactic acid production, ATP levels and glucose uptake were all significantly increased after EFNA3 overexpression in HCC827 cells. These results suggest that EFNA3 could regulate the glycolytic capacity of LUAD cells.

\section{Correlation analysis between EFNA3 expression and microenvironment}

Next, we assessed the correlation between EFNA3 expression and the tumor immune microenvironment. As shown in Fig. 9A, the expression of EFNA3 was negatively correlated with immune scores $(R=-0.28$, $\mathrm{p}<0.001)$, stromal scores $(\mathrm{R}=-0.25, \mathrm{p}<0.001)$ and ESTIMATE scores $(\mathrm{R}=-0.29, \mathrm{p}<0.001)$. The relationships between the expression of EFNA3 and tumor-infiltrating lymphocytes (TILs) were analyzed by Spearman correlation using the TISIDB database (Fig. 9B). The results indicated a negative correlation between most TILs and EFNA3 levels. As shown in Fig. 9C, the TILs display moderate correlation $(\mathrm{R}<-0.3)$ including effector memory CD4 T cell (rho $=-0.34, \mathrm{p}=2.64 \mathrm{e}-15)$, effector memory CD8 $\mathrm{T}$ cell $(\mathrm{rho}=-0.313, \mathrm{p}=4.6 \mathrm{e}-13$ ), Immature B cell (Imm B cell) (rho $=-$ 0.314, $\mathrm{p}=3.58 \mathrm{e}-13)$, NK cell $(\mathrm{rho}=-0.325, \mathrm{p}=4.67 \mathrm{e}-14)$, Eosinophil cell $(\mathrm{rho}=-0.426, \mathrm{p}=2.2 \mathrm{e}-16)$, and mast cell $(\mathrm{rho}=-0.301, \mathrm{p}=3.45 \mathrm{e}-12)$.

\footnotetext{
(See figure on next page.)

Fig. 8 EFNA3 regulate the proliferation ability and glycolytic capacity of A549 cells. mRNA (A) and protein (B) expression levels of EFNA3 in human bronchial epithelial cells (HBE) and lung adenocarcinoma cells (H1299, H1975, A549, PC9, and HCC827). C EFNA3 expression levels were detected by qRT-PCR (left) and western blotting (right) analyses after transfection with negative control (NC) or EFNA3-siRNA; D Cell viability assays showed that EFNA3 knockdown decreased A549 cell proliferation; E The number of colonies formed by A549 cells transfected with EFNA3-siRNA was lower than that for cells transfected with NC. F Lactate production, ATP level, and glucose uptake were measured in A549 cells after transfection with negative control (NC) or EFNA3-siRNA. G EFNA3 expression levels were detected by qRT-PCR (left) and western blotting (right) analyses after transfection with negative control (pcDNA3.1-NC) or pcDNA3.1-EFNA3; H Cell viability assays showed that EFNA3 overexpression increased HCC827 cell proliferation; I The number of colonies formed by HCC827 cells transfected with pcDNA3.1-EFNA3was higher than that for cells transfected with pcDNA3.1-NC; $\mathbf{J}$ Lactate production, ATP level, and glucose uptake were measured in HCC827 cells after transfection with negative control (pcDNA3.1-NC) or pcDNA3.1-EFNA3. mean \pm SD; ${ }^{*} p<0.05,{ }^{* *} p<0.01$, and ${ }^{* *} p<0.001$ (vs. control group)
} 
A

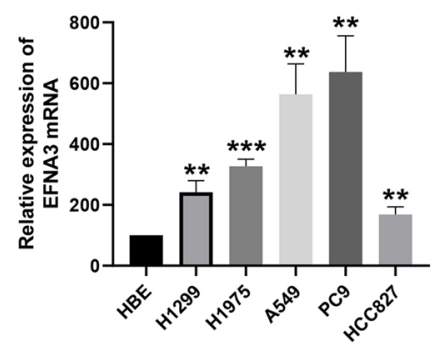

C

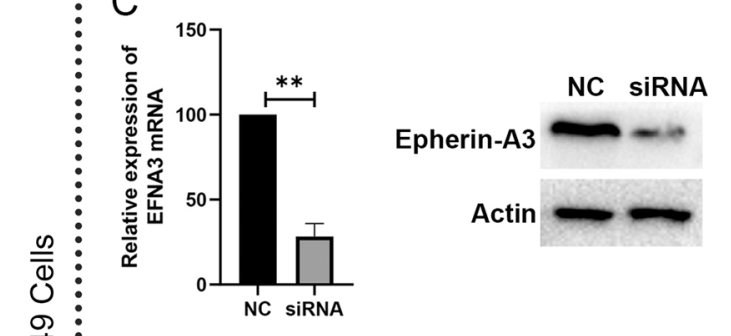

B

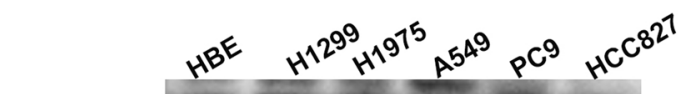

Epherin-A3

Actin

in

D

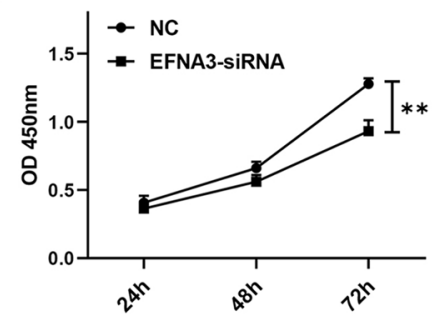

F
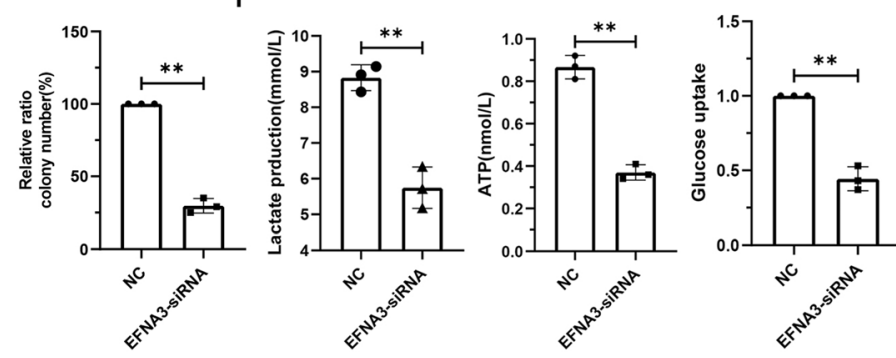

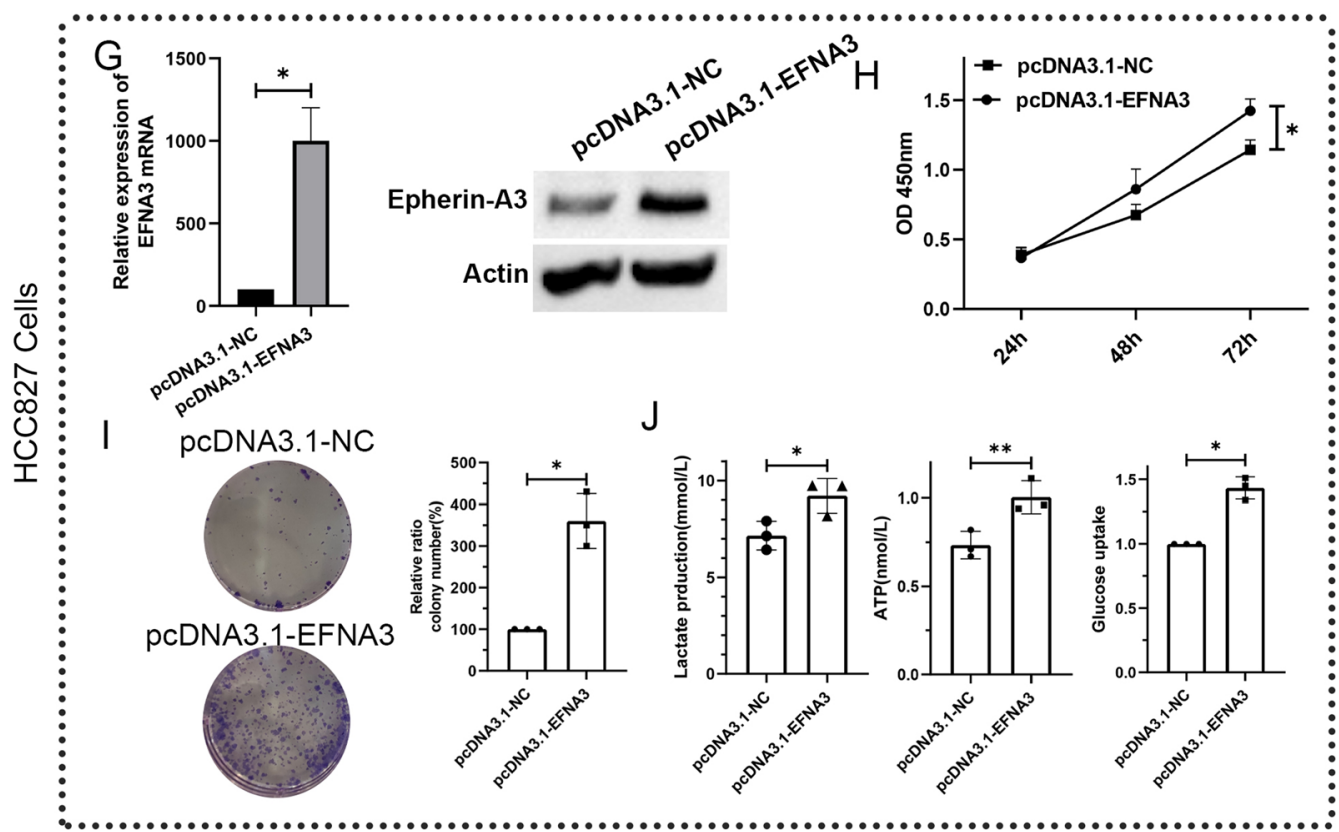

Fig. 8 (See legend on previous page.) 

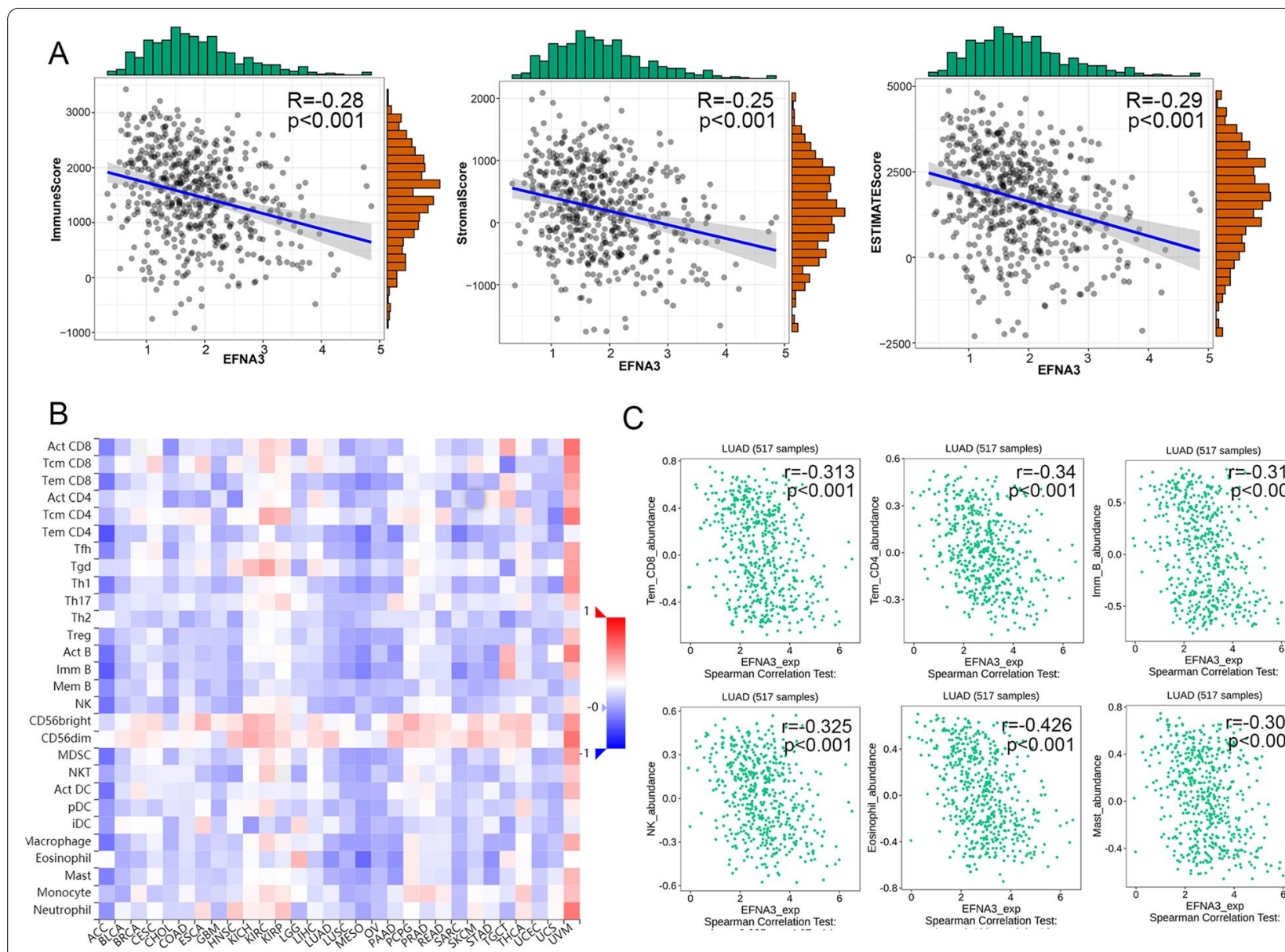

C
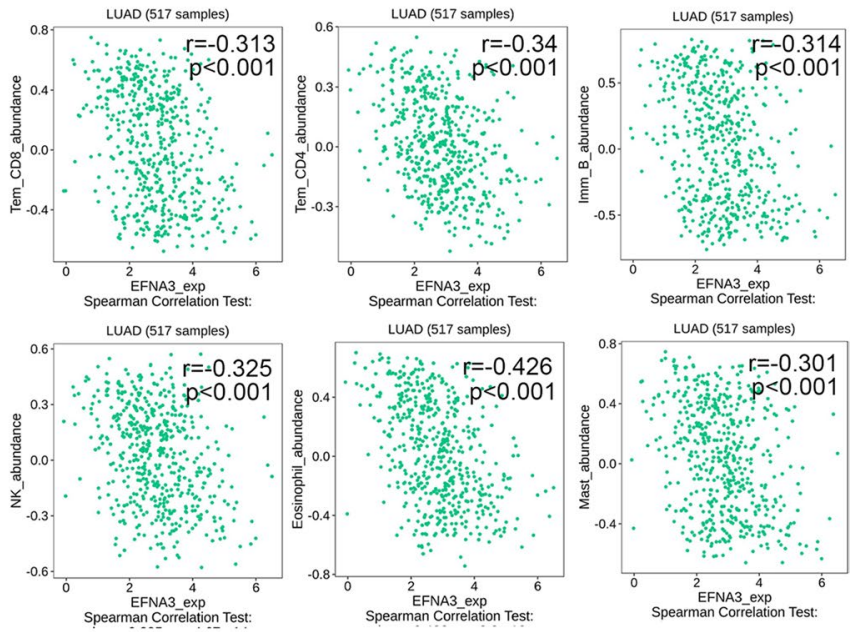

Fig. 9 Correlation analysis between EFNA3 expression and immune infiltration. A The relationship between EFNA3 expression and immune scores, stromal scores, and ESTIMATE score; $\mathbf{B}$ The abundances of TILs correlated with EFNA3 expression. C Effector memory CD4 T cell (rho $=-0.34$, $p=2.64 e-15)$, effector memory CD8 T cell (rho $=-0.313, p=4.6 e-13)$, Immature B cell (Imm B cell) (rho $=-0.314, p=3.58 e-13)$, NK cell ( $r h o=-0.325, p=4.67 e-14)$, Eosinophil cell (rho $=-0.426, p=2.2 e-16)$, and mast cell ( $r h o=-0.301, p=3.45 e-12$ ) was correlate with EFNA3 expression level in LUAD

\section{Association of EFNA3 and immunotherapy response in patients with LUAD}

Presently, immunotherapy is considered an important treatment for patients with LUAD. Therefore, we further assessed the association of EFNA3 and immunotherapy response by analyzing the correlation of EFNA3 expression and widely recognized immunotherapy biomarkers. In total, we enrolled seven indices, including $\mathrm{TMB}$, the number of neoantigens, the number of clonal neoantigens, the number of subclonal neoantigens, the TIDE score, the $\mathrm{T}$ cell dysfunction score, and the $\mathrm{T}$ cell exclusion score. As depicted in Fig. 10A-H, patients in the EFNA3-high group were distinguished by a high level of TMB, neoantigens, and $\mathrm{T}$ cell exclusion scores and low levels of TIDE and T cell dysfunction scores. These results indicate that patients with higher expression of EFNA3 may benefit from immunotherapy.

\section{Discussion}

Our research group aimed to study correlations between Eph/Ephrin family members and lung cancer. In this study, EFNA3, EFNB1, EFNB2, and EPHB2 showed a significant correlation with OS and PFS in LUAD patients and were differentially expressed in tumor tissues and normal lung tissues. EPHB2, as an EPHB subgroup receptor kinase, could modulate the biological behavior of small cell lung carcinoma through autocrine and/or juxtracrine activation by ephrin-B ligands (EFNB1, EFNB2, and EFNB3) that are expressed in the same or neighboring cells [28]. In addition, Zhao et al. reported that high expression of EPHB2 can predict poor overall survival and a high mortality rate and that it is an independent prognostic biomarker in lung adenocarcinoma patients [29]. Recent data have suggested that curcumin could regulate EPHB2 by suppressing Sp-1 activation [30]. The gene expression of EFNA3 was upregulated in early-stage 

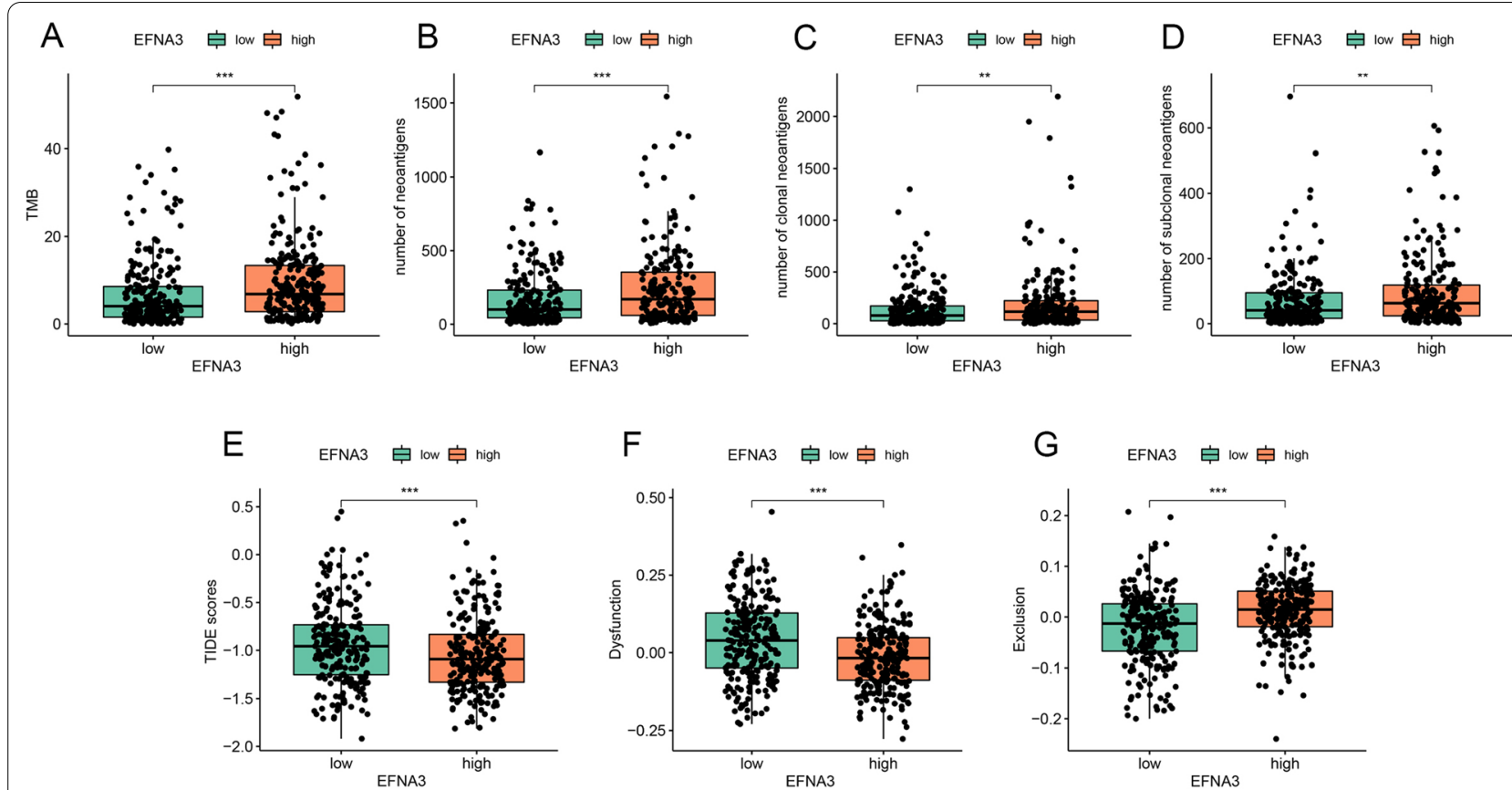

Fig. 10 The expression pattern of immunotherapy response makers in high- and low-EFNA3 groups. The distribution of TMB (A), number of neoantigens $(\mathbf{B})$, number of clonal neoantigens $(\mathbf{C})$, number of subclonal neoantigens $(\mathbf{D})$, TIDE score $(\mathbf{E})$, T cell dysfunction score $(\mathbf{F})$, T cell exclusion score (G) in EFNA3-high and EFNA3-low groups; ${ }^{*} p<0.05$, ${ }^{* *} p<0.01$, and ${ }^{* * *} p<0.001$ (vs. control group)

NSCLC [31]. Recent data [32] suggested that EFNA3 was selectively downregulated in tumor samples of lung cancer patients with COPD due to higher expression of miR210. Therefore, we believe that EFNA3, EFNB1, EFNB2, and EPHB2 are closely associated with the tumorigenesis and progression of LUAD.

The present study further focused on EFNA3, which had both significant differential expression and the most significant prognostic value. Studies have found that the expression levels of EFNA3 are inhibited in the process of skeletal muscle satellite cell formation [33]. Another study showed that EFNA3 promotes the proliferation and invasion of peripheral nerve sheath tumor cells and is regulated by miR-210 [34]. Importantly, several studies have shown that EFNA3 is involved in tumor angiogenesis [34, 35]. However, the functions of EFNA3 in the pathogenesis and progression of LUAD are still unclear. The results from the cBioPortal database suggest that amplification may be one of the main mechanisms by which EFNA3 is overexpressed in LUAD. In this study, the expression level and prognostic value were further verified in LUAD through clinical samples. The protein expression of EFNA3 was upregulated in LUAD tissue compared to normal lung tissue. In addition, the expression of the EFNA3 protein was significantly related to clinicopathological characteristics. Thus, EFNA3 gene expression is an independent prognostic risk biomarker.
According to previous studies, overexpression of EFNA3 can reduce glutamate transporter levels in astrocytes, and EphA4/EFNA3 signaling can regulate synaptic function and plasticity [36]. In addition, it has been confirmed that EFNA3 could regulate the EMT process by the PI3K/AKT signaling pathway in oral cancer [37]. In this study, purple modules related to EFNA3 were screened out through WGCNA. Further enrichment analysis showed that EFNA3 is closely related to nuclear division, synaptic function, and ion channel activity. In addition, GSEA showed that "cholesterol homeostasis," "glycolysis," and "oxidative phosphorylation" were enriched in the EFNA3-high expression group, suggesting that $E F N A 3$ may be closely related to the metabolic ability of lung adenocarcinoma cells. In short, EFNA3 may promote the malignant progression of LUAD through these potential pathways. Further experiments showed that EFNA3 knockdown could decrease the proliferation ability and glycolytic capacity of LUAD cells in vitro. Our research results suggest a potential oncogenic effect of EFNA3 in LUAD, but further research is needed to provide conclusive results. in particular, the specific oncogenic mechanism still needs further exploration.

In recent years, immunotherapy involving checkpoint inhibitors of the PD1/PDL1 axis [38, 39] has been used in clinical therapy for LUAD patients. In this study, we 
found that TMB, neoantigens, and TIDE scores were associated with patients in the EFNA3-high group. TMB is one of the classic biomarkers for immunotherapy response, and neoantigen burden is always increased by TMB $[24,40]$. The TIDE score is a newly developed method for immunotherapy response prediction and is considered a more accurate biomarker than TMB [21]. Collectively, we preliminarily speculate that EFNA3-high patients may be suitable for immunotherapy. This might be due to ephrin ligands controlling cell interactions during normal development and playing a vital role in both locally and systemically induced immune responses [41, 42]. Importantly, we found that EFNA3 was associated with the tumor microenvironment and lymphocytes. These findings give us additional confidence that EFNA3 expression may act as a novel predictive biomarker for immunotherapy response. Prospective studies should be used to further determine the relationship between EFNA3 and immunotherapy response in the future.

Future research should focus on the regulatory mechanism of high EFNA3 expression in LUAD tissues. Several studies have confirmed that posttranscriptional modification plays an important role in regulating protein expression [43, 44]. MicroRNAs (miRNAs) are a family of posttranscriptional gene repressors and have been widely associated with the regulation of gene expression in various contexts [45]. Previous studies indicated that miR210 could regulate the expression of EFNA3 by binding to its 3'-UTR in pancreatic cancer cells [46], peripheral nerve sheath tumor cells [47], oral cancer cells [37], etc. Moreover, one study demonstrated that miR-210 can regulate the expression of EFNA3 to promote sensory axon regeneration in adult mice [48]. Importantly, several studies indicate that miRNA-210 from extracellular vesicles could regulate the expression of EFNA3 to promote angiogenesis in ischemic hearts [49], oral squamous cell carcinoma [34], acute myocardial infarction [49], and ischemic disease models [50]. These studies suggest that abnormal expression of miR-210 is a possible cause of high EFNA3 expression in LUAD. Therefore, future experimental studies should be performed to explore the regulatory mechanism between miR-210 and EFNA3 in LUAD.

However, this study has some limitations. Our data indicate thatproveEFNA3 plays a role in promoting LUAD cells and could regulate glycolytic capacity, the underlying mechanism by which EFNA3 regulates glycolysis has not been elucidated. Second, because the mRNA expression data from patients with immunotherapy were not available, the prediction ability of EFNA3 for immunotherapy response was estimated indirectly. These experimental studies will be performed and reported in the future.
In conclusion, this study revealed that increased EFNA3 in LUAD patients predicted worse clinical prognosis, promoted LUAD cell proliferation and glycolysis ability, and was related to the immunotherapy response.

\begin{abstract}
Acknowledgements
Not applicable.

Authors' contributions

Conception and design: GH; Provision of study materials or patients: ZZ and TW; Collection and assembly of data: MD and RT; Data analysis and interpretation: MD, CL and XZ; Manuscript writing: All authors; Final approval of manuscript: All authors.

\section{Funding}

This research was supported by the Non-profit Central Research Institute Fund of Chinese Academy of Medical Sciences (No. 2020-PT320-001), National Natural Science Foundation of China (No. 81900040), Liaoning Education Ministry Supporting Foundation (No. QN2019014), and Liaoning Science and Technology Ministry Supporting Foundation (No.2019-ZD-0766).
\end{abstract}

Availability of data and materials

Not applicable.

\section{Declarations}

\section{Ethics approval and consent to participate}

This study was approved by the Clinical Research Ethics Committee of Shengjing Hospital Affiliated to China Medical University.

\section{Consent for publication}

Not applicable.

\section{Competing interests}

The authors declare that they have no competing interests.

\section{Author details}

${ }^{1}$ Department of Pulmonary and Critical Care Medicine, Center of Respiratory Medicine, China-Japan Friendship Hospital, Beijing 100029, China. ${ }^{2}$ Graduate School of Peking Union Medical College, Chinese Academy of Medical Sciences, Peking Union Medical College, Beijing 100029, China. ${ }^{3}$ National Center for Respiratory Medicine, Beijing 100029, China. ${ }^{4}$ Institute of Respiratory Medicine, Chinese Academy of Medical Sciences, Beijing 100029, China. ${ }^{5}$ National Clinical Research Center for Respiratory Diseases, Beijing 100029, China. ${ }^{6}$ Department of Pathology, Shengjing Hospital of China Medical University, Shenyang 110001, China. ${ }^{7}$ Department of Pathology, Shenyang KingMed Center for Clinical Laboratory Co., Ltd., Shenyang 110001, China. ${ }^{8}$ Department of Pulmonary and Critical Care Medicine, First Hospital of China Medical University, Shenyang 110001, China. ${ }^{9}$ Department of Pulmonary and Critical Care Medicine, Fourth Hospital of China Medical University, Shenyang 110001, China.

Received: 10 August 2021 Accepted: 24 September 2021

Published online: 13 October 2021

\section{References}

1. Bray F, Ferlay J, Soerjomataram I, Siegel RL, Torre LA, Jemal A. Global cancer statistics 2018: GLOBOCAN estimates of incidence and mortality worldwide for 36 cancers in 185 countries. CA Cancer J Clin. 2018;68:394-424.

2. Deng M, Liu B, Zhang Z, Chen Y, Wang Y, Wang X, et al. Loss of G-proteinsignaling modulator 2 accelerates proliferation of lung adenocarcinoma via EGFR signaling pathway. Int J Biochem Cell Biol. 2020;122:105716.

3. Hirsch FR, Scagliotti GV, Mulshine JL, Kwon R, Curran WJ, Wu Y-L, et al. Lung cancer: current therapies and new targeted treatments. Lancet (London, England). 2017;389:299-311. 
4. Miller KD, Siegel RL, Lin CC, Mariotto AB, Kramer JL, Rowland JH, et al. Cancer treatment and survivorship statistics, 2016. CA Cancer J Clin. 2016:66:271-89.

5. Pasquale EB. Eph receptor signalling casts a wide net on cell behaviour. Nat Rev Mol Cell Biol. 2005;6:462-75.

6. Miao H, Wang B. Eph/ephrin signaling in epithelial development and homeostasis. Int J Biochem Cell Biol. 2009;41:762-70.

7. Pasquale EB. Eph-ephrin bidirectional signaling in physiology and disease. Cell. 2008:133:38-52.

8. Poliakov A, Cotrina M, Wilkinson DG. Diverse roles of eph receptors and ephrins in the regulation of cell migration and tissue assembly. Dev Cell. 2004;7:465-80.

9. Eph Nomenclature Committee. Unified nomenclature for Eph family receptors and their ligands, the ephrins. Cell. 1997;90:403-4.

10. Saha N, Robev D, Mason EO, Himanen JP, Nikolov DB. Therapeutic potential of targeting the Eph/ephrin signaling complex. Int J Biochem Cell Biol. 2018;105:123-33.

11. Li J, Sun Y, Wang $X$, Wang J, Zhu Y. The expressions of EphB4 and ephrinB2 in lung adenocarcinomas: a high level of the EphB4 protein is associated with lymph node metastasis. Int J Clin Exp Pathol. 2019;12:3447-52.

12. Oweida A, Bhatia S, Hirsch K, Calame D, Griego A, Keysar S, et al. Ephrin-B2 overexpression predicts for poor prognosis and response to therapy in solid tumors. Mol Carcinog. 2017;56:1189-96.

13. Ishikawa M, Miyahara R, Sonobe M, Horiuchi M, Mennju T, Nakayama E, et al. Higher expression of EphA2 and ephrin-A1 is related to favorable clinicopathological features in pathological stage I non-small cell lung carcinoma. Lung cancer (Amsterdam, Netherlands). 2012;76:431-8.

14. Rhodes DR, Kalyana-Sundaram S, Mahavisno V, Varambally R, Yu J, Briggs BB, et al. Oncomine 3.0: genes, pathways, and networks in a collection of 18,000 cancer gene expression profiles. Neoplasia (New York, NY). 2007;9:166-80.

15. Tang Z, Li C, Kang B, Gao G, Li C, Zhang Z. GEPIA: a web server for cancer and normal gene expression profiling and interactive analyses. Nucleic Acids Res. 2017:45:w98-W102.

16. Győrffy B, Surowiak P, Budczies J, Lánczky A. Online survival analysis software to assess the prognostic value of biomarkers using transcriptomic data in non-small-cell lung cancer. PLoS ONE. 2013;8:e82241.

17. Gao J, Aksoy BA, Dogrusoz U, Dresdner G, Gross B, Sumer SO, et al. Integrative analysis of complex cancer genomics and clinical profiles using the cBioPortal. Sci Signal. 2013;6:pl1.

18. Deng M, Liu B, Zhang Z, Chen Y, Wang Y, Wang X, et al. Knockdown of G-protein-signaling modulator 2 promotes metastasis of non-small-cell lung cancer by inducing the expression of Snail. Cancer Sci. 2020;111:3210-21.

19. Deng M, Yu R, Wang S, Zhang Y, Li Z, Song H, et al. Limb-bud and heart attenuates growth and invasion of human lung adenocarcinoma cells and predicts survival outcome. Cell Physiol Biochem. 2018;47:223-34.

20. Charoentong P, Finotello F, Angelova M, Mayer C, Efremova M, Rieder D, et al. Pan-cancer immunogenomic analyses reveal genotype-immunophenotype relationships and predictors of response to checkpoint blockade. Cell Rep. 2017;18:248-62

21. Jiang P, Gu S, Pan D, Fu J, Sahu A, Hu X, et al. Signatures of T cell dysfunction and exclusion predict cancer immunotherapy response. Nat Med. 2018;24:1550-8

22. Li B, Cui Y, Diehn M, Li R. Development and validation of an individualized immune prognostic signature in early-stage nonsquamous non-small cell lung cancer. JAMA Oncol. 2017:3:1529-37.

23. Imielinski M, Berger AH, Hammerman PS, Hernandez B, Pugh TJ, Hodis E, et al. Mapping the hallmarks of lung adenocarcinoma with massively parallel sequencing. Cell. 2012;150:1107-20.

24. Rizvi NA, Hellmann MD, Snyder A, Kvistborg P, Makarov V, Havel JJ, et al. Cancer immunology Mutational landscape determines sensitivity to PD-1 blockade in non-small cell lung cancer. Science (New York, NY). 2015;348:124-8.

25. Chen J, Yang H, Teo ASM, Amer LB, Sherbaf FG, Tan CQ, et al. Genomic landscape of lung adenocarcinoma in East Asians. Nat Genet. 2020;52:177-86.

26. Collisson EA, Campbell J, Brooks A, Berger A, Lee W, Chmielecki J, Beer D, Cope L, Creighton C, Danilova L, Ding L. Comprehensive molecular profiling of lung adenocarcinoma. Nature. 2014;511:543-50.

27. Ding L, Getz G, Wheeler DA, Mardis ER, McLellan MD, Cibulskis K, et al. Somatic mutations affect key pathways in lung adenocarcinoma. Nature. 2008:455:1069-75.
28. Tang XX, Brodeur GM, Campling BG, Ikegaki N. Coexpression of transcripts encoding $\mathrm{EPHB}$ receptor protein tyrosine kinases and their ephrin-B ligands in human small cell lung carcinoma. Clin Cancer Res. 1999;5:455-60.

29. Zhao C, Wang A, Lu F, Chen H, Fu P, Zhao X, et al. Overexpression of junctional adhesion molecule-A and EphB2 predicts poor survival in lung adenocarcinoma patients. Tumour Biol J Int Soc Oncodevelopmental Biol Med. 2017:39:1010428317691000.

30. Vallianou NG, Evangelopoulos A, Schizas N, Kazazis C. Potential anticancer properties and mechanisms of action of curcumin. Anticancer Res. 2015:35:645-51.

31. Metodieva SN, Nikolova DN, Cherneva RV, Dimova II, Petrov DB, Toncheva DI. Expression analysis of angiogenesis-related genes in Bulgarian patients with early-stage non-small cell lung cancer. Tumori. 2011;97:86-94.

32. Mateu-Jimenez M, Curull V, Rodríguez-Fuster A, Aguiló R, Sánchez-Font A, Pijuan $\mathrm{L}$, et al. Profile of epigenetic mechanisms in lung tumors of patients with underlying chronic respiratory conditions. Clin Epigenetics. 2018;10:7.

33. Alonso-Martin S, Rochat A, Mademtzoglou D, Morais J, de Reyniès A, Auradé $F$, et al. Gene expression profiling of muscle stem cells identifies novel regulators of postnatal myogenesis. Front Cell Dev Biol. 2016;4:58.

34. Wang H, Wang L, Zhou X, Luo X, Liu K, Jiang E, et al. OSCC Exosomes regulate miR-210-3p targeting EFNA3 to promote oral cancer angiogenesis through the PI3K JAKT pathway. Biomed Res Int. 2020;2020:2125656.

35. Bhuvaneswari R, Gan YY, Lucky SS, Chin WWL, Ali SM, Soo KC, et al. Molecular profiling of angiogenesis in hypericin mediated photodynamic therapy. Mol Cancer. 2008;7:56

36. Filosa A, Paixão S, Honsek SD, Carmona MA, Becker L, Feddersen B, et al. Neuron-glia communication via EphA4/ephrin-A3 modulates LTP through glial glutamate transport. Nat Neurosci. 2009;12:1285-92.

37. Wang L, Song Y, Wang H, Liu K, Shao Z, Shang Z. MiR-210-3p-EphrinA3PI3KJAKT axis regulates the progression of oral cancer. J Cell Mol Med. 2020:24:4011-22.

38. Sacher AG, Gandhi L. Biomarkers for the clinical use of PD-1/PD-L1 inhibitors in non-small-cell lung cancer: a review. JAMA Oncol. 2016;2:1217-22.

39. Arbour KC, Riely GJ. Systemic therapy for locally advanced and metastatic non-small cell lung cancer: a review. JAMA. 2019;322:764-74.

40. Gong Z, Jia Q, Chen J, Diao X, Gao J, Wang X, et al. Impaired cytolytic activity and loss of clonal neoantigens in elderly patients with lung adenocarcinoma. J Thoracic Oncol. 2019;14:857-66.

41. Shiuan $\mathrm{E}$, Chen J. Eph receptor tyrosine kinases in tumor immunity. Can Res. 2016:76:6452-7.

42. Darling TK, Lamb TJ. Emerging roles for Eph receptors and Ephrin ligands in immunity. Front Immunol. 2019:10:1473.

43. Lin S, Gregory RI. MicroRNA biogenesis pathways in cancer. Nat Rev Cancer. 2015;15:321-33.

44. Rupaimoole R, Calin GA, Lopez-Berestein G, Sood AK. miRNA deregulation in cancer cells and the tumor microenvironment. Cancer Discov. 2016:6:235-46.

45. Agbu P, Carthew RW. MicroRNA-mediated regulation of glucose and lipid metabolism. Nat Rev Mol Cell Biol. 2021:22:425-38.

46. Chen W-Y, Liu W-J, Zhao Y-P, Zhou L, Zhang T-P, Chen G, et al. Induction, modulation and potential targets of miR-210 in pancreatic cancer cells. HBPD Int. 2012;11:319-24.

47. Wang Z, Yin B, Wang B, Ma Z, Liu W, Lv G. MicroRNA-210 promotes proliferation and invasion of peripheral nerve sheath tumor cells targeting EFNA3. Oncol Res. 2013;21:145-54.

48. Hu Y-W, Jiang J-J, Yan G, Wang R-Y, Tu G-J. MicroRNA-210 promotes sensory axon regeneration of adult mice in vivo and in vitro. Neurosci Lett. 2016;622:61-6.

49. Song B-W, Lee CY, Kim R, Kim WJ, Lee HW, Lee MY, et al. Multiplexed targeting of miRNA-210 in stem cell-derived extracellular vesicles promotes selective regeneration in ischemic hearts. Exp Mol Med. 2021;53:695-708.

50. Besnier M, Gasparino S, Vono R, Sangalli E, Facoetti A, Bollati V, et al. miR-210 Enhances the Therapeutic Potential of Bone-Marrow-Derived Circulating Proangiogenic Cells in the Setting of Limb Ischemia. Mol Ther. 2018;26:1694-705

\section{Publisher's Note}

Springer Nature remains neutral with regard to jurisdictional claims in published maps and institutional affiliations. 